\title{
ASYMPTOTIC SHAPE FOR THE CHEMICAL DISTANCE AND FIRST-PASSAGE PERCOLATION ON THE INFINITE BERNOULLI CLUSTER
}

\author{
Olivier Garet ${ }^{1}$ and RÉGine Marchand ${ }^{2}$
}

\begin{abstract}
The aim of this paper is to extend the well-known asymptotic shape result for first-passage percolation on $\mathbb{Z}^{d}$ to first-passage percolation on a random environment given by the infinite cluster of a supercritical Bernoulli percolation model. We prove the convergence of the renormalized set of wet vertices to a deterministic shape that does not depend on the realization of the infinite cluster. As a special case of our result, we obtain an asymptotic shape theorem for the chemical distance in supercritical Bernoulli percolation. We also prove a flat edge result in the case of dimension 2. Various examples are also given.
\end{abstract}

Mathematics Subject Classification. 60G15, 60K35, 82B43.

Received September 4, 2003. Revised June 25, 2004.

First-passage percolation was introduced by Hammersley and Welsh [12] as a model for the spread of a fluid in a porous medium. To each edge of the $\mathbb{Z}^{d}$ lattice is attached a nonnegative random variable which corresponds to the travel time needed by the fluid to cross the edge.

When the passage times are independent identically distributed variables, Cox and Durrett [7] showed that, under some moment conditions, the renormalized set of wet vertices at time $t$ almost surely converges to a deterministic asymptotic shape. Derriennic (cited by Kesten [13]), and next Boivin [3], progressively extended the result to the stationary ergodic case. Häggström and Meester [11] also proved that every symmetric compact set with nonempty interior can be obtained as the asymptotic shape of a stationary first-passage percolation model.

In this paper, we want to study the analogous problem of spread of a fluid in a more complex medium. On one hand, an edge can either be open or closed according to the local properties of the medium - e.g. the absence or the presence of non-porous particles. In other words, the $\mathbb{Z}^{d}$ lattice is replaced by a random environment given by the infinite cluster of a super-critical Bernoulli percolation model. On the other hand, as in the classical model, a random passage time is attached to each open edge. This random time corresponds to the local porosity of the medium - e.g. the density of the porous phase. Thus, our model can be seen as a combination between classical Bernoulli percolation and stationary first-passage percolation.

Keywords and phrases. Percolation, first-passage percolation, chemical distance, infinite cluster, asymptotic shape, random environment.

${ }^{1}$ Laboratoire de Mathématiques, Applications et Physique Mathématique d'Orléans UMR 6628, Université d'Orléans, BP 6759, 45067 Orléans Cedex 2, France; e-mail: Olivier.Garet@labomath.univ-orleans.fr

2 Institut Elie Cartan Nancy (mathématiques), Université Henri Poincaré Nancy 1, Campus Scientifique, BP 239, 54506 Vandœuvre-lès-Nancy Cedex, France; e-mail: Regine.Marchand@iecn.u-nancy.fr 
Our aim is to prove in this context the convergence of the renormalized set of wet vertices to a deterministic shape (Th. 5.3) which does not depend on the random environment. As a special case of our result, we obtain an asymptotic shape theorem for the chemical distance in supercritical Bernoulli percolation.

In the first section, we give some notations adapted to this problem, clarify the assumptions on the passage time and introduce the background of ergodic theory needed for the main proof. In Section 2, we give some estimates on the chemical distance and the travel times. In Section 3, we prove the existence of an asymptotic time constant in a given direction and study its properties (such as continuity, homogeneity and sub-additivity...). Section 4 is devoted to the question of the finiteness of these time constants, and in Section 5 we prove the asymptotic shape result. In Section 6, we study the existence of a flat edge in the asymptotic shape. Finally, in the last section, we develop some examples, such as exponential times, correlated chi-square passage times, and a model for road networks.

\section{Notations, DEFINITIONS AND PRELIMINARY LEMMAS}

\section{The random environment}

Let us first construct a Bernoulli percolation structure on $\mathbb{Z}^{d}$. Consider the graph whose vertices are the points of $\mathbb{Z}^{d}$, and put a non-oriented edge between each pair $\{x, y\}$ of vertices in $\mathbb{Z}^{d}$ such that the Euclidean distance between $x$ and $y$ is equal to 1 . Two such vertices are called neighbours. This set of edges is denoted by $\mathbb{E}^{d}$.

Set $\Omega_{E}=\{0,1\}^{\mathbb{E}^{d}}$. In the whole paper, $p$ is assumed to satisfy to

$$
p \in\left(p_{c}, 1\right]
$$

where $p_{c}=p_{c}(d)$ is the critical probability for bond percolation on $\mathbb{Z}^{d}$. We denote by $\mathbb{P}_{p}$ the product probability $\left(p \delta_{1}+(1-p) \delta_{0}\right)^{\otimes \mathbb{E}^{d}}$.

A point $\omega$ in $\Omega_{E}$ is a random environment for the first passage percolation. An edge $e \in \mathbb{E}^{d}$ is said to be open in the environment $\omega$ if $\omega(e)=1$, and closed otherwise. The states of the different edges are thus independent under $\mathbb{P}_{p}$.

A path is a sequence $\gamma=\left(x_{1}, e_{1}, x_{2}, e_{2}, \ldots, x_{n}, e_{n}, x_{n+1}\right)$ such that $x_{i}$ and $x_{i+1}$ are neighbours and $e_{i}$ is the edge between $x_{i}$ and $x_{i+1}$. We will also sometimes describe $\gamma$ only by its vertices $\gamma=\left(x_{1}, x_{2}, \ldots, x_{n}, x_{n+1}\right)$ or by its edges $\gamma=\left(e_{1}, e_{2}, \ldots, e_{n}\right)$. The number $n$ of edges in $\gamma$ is called the length of $\gamma$ and is denoted by $|\gamma|$. We will actually only consider simple paths for which the visited vertices are all distinct. A path is said to be open in the environment $\omega$ if all its edges are open in $\omega$.

The clusters of a environment $\omega$ are the connected components of the graph induced on $\mathbb{Z}^{d}$ by the open edges in $\omega$. For $x$ in $\mathbb{Z}^{d}$, we denote by $C(x)$ the cluster containing $x$. In other words, $C(x)$ is the set of vertices in $\mathbb{Z}^{d}$ that are linked to $x$ by an open path. We note $x \leftrightarrow y$ to signify that $x$ and $y$ belong to the same cluster. We denote by $C_{\infty}$ the random set: $C_{\infty}=\left\{x \in \mathbb{Z}^{d} ;|C(x)|=+\infty\right\}$. As for $p>p_{c}$, there exists almost surely one unique infinite cluster, $C_{\infty}$ is almost surely connected - see Aizenman, Kesten and Newman [1], and Burton and Keane [5] for the uniqueness result.

Moreover, for $p>p_{c}$, we have $\mathbb{P}_{p}\left(0 \in C_{\infty}\right)>0$. Since the fluid only uses open edges to travel through the medium, we will only study its behaviour on the infinite cluster. Thus we introduce the conditional probability measure $\overline{\mathbb{P}}_{p}$ on $\Omega_{E}$ by

$$
\overline{\mathbb{P}}_{p}(A)=\frac{\mathbb{P}_{p}\left(A \cap\left\{0 \in C_{\infty}\right\}\right)}{\mathbb{P}_{p}\left(0 \in C_{\infty}\right)}
$$

\section{The passage times}

We now give to each edge of the graph $\mathbb{Z}^{d}$ a passage time, which represents the time needed by a fluid to cross the edge. Consider thus $\Omega_{S}=\mathbb{R}_{+}^{\mathbb{E}^{d}}$, and a probability measure $S_{\nu}$ on $\Omega_{S}$. We use the abusive notation $S_{\nu}$ 
to help the reader keep in mind the case of a product measure $S_{\nu}=\nu^{\otimes \mathbb{E}^{d}}$, although we will consider the weaker assumption that $S_{\nu}$ is a stationary ergodic probability measure - see below for a precise definition.

\section{The probability space}

Consequently, we take $\Omega=\Omega_{E} \times \Omega_{S}$ as probability space. A point in $\Omega$ is denoted by $(\omega, \eta)$, with $\omega \in \Omega_{E}$ and $\eta \in \Omega_{S}$. We endow $\Omega$ with the probability $\mathbb{P}=\mathbb{P}_{p} \otimes S_{\nu}$ and its conditional version:

$$
\overline{\mathbb{P}}=\overline{\mathbb{P}}_{p} \otimes S_{\nu}
$$

\section{The two distances and the main result}

We first introduce the chemical distance $D(x, y)(\omega)$ between $x$ and $y$ in $\mathbb{Z}^{d}$ :

$$
D(x, y)(\omega)=\inf _{\gamma}|\gamma|,
$$

where the infimum is taken on the set of paths whose extremities are $x$ and $y$ and that are open in the environment $\omega$. By convention, $D(x, y)=+\infty$ when $x$ and $y$ are not in the same percolation cluster. The distance $D(x, y)$ is thus, when it is finite, the minimal number of open edges needed to link $x$ and $y$ in the environment $\omega$. Note that $D(x, y)$ only depends on the random environment $\omega$. We denote by $\gamma(x, y)$ an open path between $x$ and $y$ with this minimal number of edges. When necessary, we can define uniquely $\gamma(x, y)$ by choosing an order on the set of edges $\mathbb{E}^{d}$ and taking the minimal such path for the lexical order on the edges of the path.

For $(\omega, \eta) \in \Omega$, and $(x, y) \in \mathbb{Z}^{d} \times \mathbb{Z}^{d}$, we define the travel time $d(x, y)(\omega, \eta)$ to be

$$
\inf _{\gamma} \sum_{e \in \gamma} \eta_{e}
$$

where the infimum is taken on the set of paths whose extremities are $x$ and $y$ and that are open in the environment $\omega$. Of course $d(x, y)=+\infty$ if and only if $D(x, y)=+\infty$. It follows that the event $\{d(x, y)<+\infty\}$ only depends on the random environment $\omega$. For $t \geq 0$, we note

$$
B_{t}=\left\{k \in \mathbb{Z}^{d} ; d(0, k) \leq t\right\} .
$$

This is the random set of vertices that can be reached from the origin in a time smaller than $t$; note that it is a non-decreasing random set in $t$. The main goal of this paper is to prove the following shape theorem (see Sect. 5 for precise conditions and statement):

Main result. Under some mild conditions, there exists a norm $\mu$ such that

$$
\lim _{t \rightarrow+\infty} \mathcal{D}\left(\frac{B_{t}}{t}, B_{\mu}\right)=0 \quad \overline{\mathbb{P}} \text { a.s. }
$$

where $B_{\mu}$ is the unit ball for the norm $\mu$, and $\mathcal{D}$ denotes the Hausdorff distance between two compact subsets of $\mathbb{R}^{d}$.

Remarks. 1. A natural approach of this result could be to consider the continuity property of the time constant in the first axis direction (see Cox [6] and Cox and Kesten [8]). In the case of first-passage percolation with independent identically distributed passage times, the continuity result says that if the distribution functions $\left(F_{n}\right)$ of the passage time weakly converge to a distribution $F$, then the time constants $\left(\mu_{F_{n}}\right)$ along the first coordinate axis converge to the time constant $\mu_{F}$.

In our context, consider independent identically distributed passage times $t(e)$ with common distribution function $F$ on the infinite cluster of Bernoulli percolation with parameter $p>p_{c}$. We can consider the following 
sequence of approximate passage times:

$$
t_{n}(e)=t(e) \mathbb{1}_{\{e \text { open }\}}+n \mathbb{1}_{\{e \text { closed }\}}
$$

with distribution function $F_{n}(x)=p F(x)+(1-p) \mathbb{1}_{[0, \infty)}(x)$, which converges to $p F$ (which is not a distribution function). In the light of the continuity result, one could conjecture that $\lim \mu_{F_{n}}$ is exactly the time constant related to $F$ in the random environment with parameter $p$. Unfortunately, it does not seem so easy to use such arguments to prove the existence of the time constant in random environment.

2. The mild conditions of the shape theorem are in fact integrability conditions (see Condition $\left(H_{\alpha}\right)$ ). In the case of independent identically distributed passage times in a random environment with $p<1$, we require a moment of order $2+\delta, \delta>0$, for the convergence of renormalized passage times to the time constant and a moment of order $2\left(d^{2}+2 d-1\right)+\delta, \delta>0$, for the shape theorem itself. These conditions are of course much stronger than in the classical case with $p=1$ (see Cox and Durrett [7]) and could probably be improved.

However, considering a random environment with $p<1$ is equivalent to consider passage times that can take the value $+\infty$ with probability $1-p>0$, and that admit thus no finite moment. Our integrability condition is in fact a moment condition on the passage time conditioned to be finite. One of the difficulties to weaken this condition is due to the fact that our knowledge of the structure of the percolating cluster does not allow to make a choice between several disjoint (short) paths joining two points, as it was the case in the classical setting.

\section{Usual definitions and results in ergodic theory}

We recall here some classical definitions, notations and results in ergodic theory which can be found in any course - see for instance the book written by Brown [4].

Consider a probability space $(\Omega, \mathcal{F}, \mathcal{Q})$. A map $\theta: \Omega \rightarrow \Omega$ is said to be a measure-preserving transformation if for each $A \in \mathcal{F}, \mathcal{Q}\left(\theta^{-1}(A)\right)=\mathcal{Q}(A)$. The quadruple $(\Omega, \mathcal{F}, \mathcal{Q}, \theta)$ is then called a dynamical system.

For any measurable set $A$, we can define the entrance time in $A$ :

$$
n_{A}(\omega)=\inf \left\{n \geq 1 ; \theta^{n} \omega \in A\right\} .
$$

The well-known recurrence theorem of Poincaré ensures that for $\mathcal{Q}$ almost every $\omega \in A,\left\{n \geq 1 ; \theta^{n} \omega \in A\right\}$ is infinite.

If every subset of $\Omega$ which is invariant by $\theta$ has probability 0 or 1 , we say that $\theta$ is ergodic for $\mathcal{Q}$. When $\theta$ is ergodic, the mean entrance time is given by Kac's formula:

$$
\int_{A} n_{A} \mathrm{~d} \mathcal{Q}=1
$$

For a set $A \in \mathcal{F}$ such that $\mathcal{Q}(A)>0$, the entrance time in $A$ induces a transformation $\theta_{A}: \Omega \rightarrow \Omega$ defined by $\theta_{A}(\omega)=\theta^{n_{A}(\omega)}(\omega)$. Of course, $\theta_{A}$ is in general only defined for almost every $\omega \in A$ - this is a consequence of the recurrence theorem - but its definition may be extended to $A$. If $\mathcal{Q}_{A}$ is defined by $\mathcal{Q}_{A}(B)=\frac{\mathcal{Q}(A \cap B)}{\mathcal{Q}(A)}$, the following results hold:

- If $\theta$ is a $\mathcal{Q}$-preserving transformation, then $\theta_{A}$ is a $\mathcal{Q}_{A}$-preserving transformation.

- If $\theta$ is ergodic for $\mathcal{Q}$, then $\theta_{A}$ is ergodic for $\mathcal{Q}_{A}$.

Note that Kac's formula can here be rewritten in the following form:

$$
\int_{\Omega} n_{A} \mathrm{~d} \mathcal{Q}_{A}=\frac{1}{\mathcal{Q}(A)}
$$




\section{The translation operators and the integrability conditions}

We introduce here the classical translation operators on $\mathbb{Z}^{d}$ and give our precise assumptions on the law of the passage time $S_{\nu}$.

Definition 1.1. For any set $X$ and any $u \in \mathbb{Z}^{d}$, we define the translation operator $\theta_{u}$ on $X^{\mathbb{E}^{d}}$ by the relation

$$
\forall e \in \mathbb{E}^{d} \quad\left(\theta_{u} \omega\right)_{e}=\omega_{u . e},
$$

where u.e denotes the natural action of $\mathbb{Z}^{d}$ on $\mathbb{E}^{d}$ : if $e=\{a, b\}$, then $u . e=\{a+u, b+u\}$.

In the whole paper, the measure $S_{\nu}$ which describes the passage time is assumed to be an ergodic measure preserving the $\mathbb{Z}^{d}$ actions $\left(\theta_{u}\right)_{u \in \mathbb{Z}^{d}}$. It means that for each $u \in \mathbb{Z}^{d},\left(\Omega_{S}, \mathcal{B}\left(\Omega_{S}\right), S_{\nu}, \theta_{u}\right)$ is an ergodic dynamical system. This covers of course the case of a product measure $S_{\nu}=\nu^{\otimes \mathbb{E}^{d}}$.

Since $S_{\nu}$ is invariant under the action of $\mathbb{Z}^{d}$, the law of the passage time of a given edge only depends on its direction. Let us denote by $\left(e_{1}, \ldots, e_{d}\right)$ the canonical basis of $\mathbb{Z}^{d}$. In the whole paper, we will assume that

$$
\left(H_{\text {int }}\right) \quad m=\sup _{1 \leq i \leq d} \int \eta_{\left\{0, e_{i}\right\}} \mathrm{d} S_{\nu}(\eta)=\sup _{e \in \mathbb{E}^{d}} \int \eta_{e} \mathrm{~d} S_{\nu}(\eta)<+\infty .
$$

When needed, we will assume that $S_{\nu}$ satisfies to

$$
\left(H_{\alpha}\right) \quad \exists A_{\alpha}, B_{\alpha}>0 \text { such that } \forall \Lambda \subseteq \mathbb{E}^{d} \quad S_{\nu}\left(\eta \in \Omega_{S} ; \sum_{e \in \Lambda} \eta_{e} \geq B_{\alpha}|\Lambda|\right) \leq \frac{A_{\alpha}}{|\Lambda|^{\alpha}},
$$

for an appropriate value of $\alpha$. Assumption $\left(H_{\alpha}\right)$ is in fact a combination between assumptions on the moments and assumptions on the dependence between the passage times of distinct edges.

For instance, if $S_{\nu}$ is the product measure $\nu^{\otimes \mathbb{E}^{d}}$, Assumption $\left(H_{\alpha}\right)$ follows from the Marcinkiewicz-Zygmund inequality as soon as the passage time of an edge has a moment of order $2 \alpha-$ see e.g. Theorem 3.7.8 in Stout [18]. Note that obviously, $\left(H_{\alpha}\right)$ is fulfilled for all $\alpha>1$ when the passage times are bounded.

Definition 1.2. For each $u \in \mathbb{Z}^{d} \backslash\{0\}$, let $T_{u}(\omega)=\inf \left\{n \geq 1 ; n u \in C_{\infty}(\omega)\right\}$ and define the random translation operator on $\Omega=\Omega_{E} \times \Omega_{S}$

$$
\Theta_{u}(\omega, \eta)=\left(\theta_{u}^{T_{u}(\omega)}(\omega), \theta_{u}^{T_{u}(\omega)}(\eta)\right) .
$$

Note that $T_{u}$ only depends on the environment $\omega$, and not on the passage times $\eta$, whereas the operator $\Theta_{u}$ acts on the whole configuration $(\omega, \eta)$. The new configuration $\Theta_{u}(\omega, \eta)$ is the initial configuration viewed from the first vertex of the form $n u$ to be in the infinite cluster.

Lemma 1.3. $\Theta_{u}$ is a $\overline{\mathbb{P}}$-preserving transformation. Moreover, $\Theta_{u}$ is ergodic for $\overline{\mathbb{P}}$ and

$$
\mathbb{E}_{\overline{\mathbb{P}}} T_{u}=\frac{1}{\mathbb{P}_{p}\left(0 \in C_{\infty}\right)} .
$$

Proof. Let $u \in \mathbb{Z}^{d}$. The dynamical system $\left(\Omega, \mathcal{B}(\Omega), \mathbb{P}, \theta_{u} \times \theta_{u}\right)$ is the direct product of the mixing system $\left(\Omega_{E}, \mathcal{B}\left(\Omega_{E}\right), \mathbb{P}_{p}, \theta_{u}\right)$ by the ergodic system $\left(\Omega_{S}, \mathcal{B}\left(\Omega_{S}\right), S_{\nu}, \theta_{u}\right)$. Since the product of a weakly mixing system and an ergodic system is ergodic - see for instance Proposition 1.6 of Brown [4] -, it follows that $\theta_{u}$ is ergodic for $\mathbb{P}$. It is easy to see that $\Theta_{u}$ is the transformation induced by $\theta_{u} \times \theta_{u}$ on the set $A=\left\{0 \in C_{\infty}\right\} \times \Omega_{S}$. Since $\mathbb{P}(A)=\mathbb{P}_{p}\left(0 \in C_{\infty}\right) S_{\nu}\left(\Omega_{S}\right)=\mathbb{P}_{p}\left(0 \in C_{\infty}\right)>0$, the announced result follows from Kac's formula.

Definition 1.4. Let us define for $n \geq 1$

$$
T_{n, u}(\omega)=\sum_{k=0}^{n-1} T_{u}\left(\Theta_{u}^{k} \omega\right)
$$


Note that $T_{n, u}$ represents the position of the $n$th intersection of the infinite cluster with the half line $\mathbb{Z}_{+}^{*} u$.

Lemma 1.5. $\frac{T_{n, u}}{n} \rightarrow \frac{1}{\mathbb{P}_{p}\left(0 \in C_{\infty}\right)} \quad \overline{\mathbb{P}}$ a.s.

Proof. Since $\Theta_{u}$ is ergodic for $\overline{\mathbb{P}}$, this immediately follows from the point-wise ergodic theorem.

\section{Estimates on the Chemical Distance AND tRAVEL Times}

The main goal of this section is to prove the integrability of the chemical distance $D\left(0, T_{1, u} u\right)$ and of the travel time $d\left(0, T_{1, u} u\right)$ in order to apply the sub-additive ergodic theorem. This is more intricate than in the classical case as the structure of the open paths is random itself. Fortunately, Antal and Pisztora's following result (Th. 1.1 in Antal and Pisztora [2]) will be a fundamental tool to control the chemical distance: if $p>p_{c}(d)$, there exist a constant $\rho=\rho(p, d) \in[1,+\infty)$ and two strictly positive constants $A_{\rho}$ and $B_{\rho}$ such that:

$$
\forall x \in \mathbb{Z}^{d}, \mathbb{P}_{p}\left(0 \leftrightarrow x, D(0, x)>\rho\|x\|_{1}\right) \leq A_{\rho} \exp \left(-B_{\rho}\|x\|_{1}\right)
$$

Remember here that the most relevant norm on $\mathbb{R}^{d}$ in the context of first passage percolation is the $l_{1}$-norm:

$$
\forall y=\left(y_{1}, \ldots, y_{d}\right) \in \mathbb{R}^{d},\|y\|_{1}=\sum_{i=1}^{d}\left|y_{i}\right|
$$

as $\|z-y\|_{1}$ represents, for two vertices $y$ and $z$ in $\mathbb{Z}^{d}$, the minimal number of edges needed to link them.

The next lemma gives, under Assumption $\left(H_{\alpha}\right)$, an analogous estimate for the travel times:

Lemma 2.1. If $\left(H_{\alpha}\right)$ holds with two positive constants $A_{\alpha}$ and $B_{\alpha}$, and if $\rho$ is the constant that appears in (1), then there exists a positive constant $C_{\alpha}$ such that

$$
\forall x \in \mathbb{Z}^{d}, \mathbb{P}\left(0 \leftrightarrow x ; d(0, x) \geq B_{\alpha} \rho\|x\|_{1}\right) \leq \frac{C_{\alpha}}{\|x\|_{1}^{\alpha}}
$$

Proof. Let $x \in \mathbb{Z}^{d}$. By definition, $d(0, x) \leq \sum_{e \in \gamma(0, x)} \eta(e)$. Then

$$
\mathbb{P}\left(0 \leftrightarrow x ; d(0, x) \geq B_{\alpha} \rho\|x\|_{1}\right) \leq \mathbb{P}\left(0 \leftrightarrow x ; \sum_{e \in \gamma(0, x)} \eta(e) \geq B_{\alpha} \rho\|x\|_{1}\right)
$$

We can now cut this probability in two pieces:

$$
\begin{aligned}
& \mathbb{P}\left(0 \leftrightarrow x ; \sum_{e \in \gamma(0, x)} \eta(e) \geq B_{\alpha} \rho\|x\|_{1}\right) \leq \mathbb{P}\left(0 \leftrightarrow x ; D(0, x) \leq \rho\|x\|_{1} ; \sum_{e \in \gamma(0, x)} \eta(e) \geq B_{\alpha} \rho\|x\|_{1}\right) \\
&+\mathbb{P}\left(0 \leftrightarrow x ; D(0, x)>\rho\|x\|_{1}\right) .
\end{aligned}
$$


Thanks to (1), we already know that the second term exponentially decreases. For the first one, we have:

$$
\begin{aligned}
\mathbb{P}\left(0 \leftrightarrow x ; D(0, x) \leq \rho\|x\|_{1} ; \sum_{e \in \gamma(0, x)} \eta(e) \geq B_{\alpha} \rho\|x\|_{1}\right) \\
=\sum_{\gamma,|\gamma| \leq \rho\|x\|_{1}} \mathbb{P}\left(0 \leftrightarrow x ; \gamma(0, x)=\gamma ; \sum_{e \in \gamma} \eta(e) \geq B_{\alpha} \rho\|x\|_{1}\right) \\
=\sum_{\gamma,|\gamma| \leq \rho\|x\|_{1}} \mathbb{P}_{p}(0 \leftrightarrow x ; \gamma(0, x)=\gamma) S_{\nu}\left(\sum_{e \in \gamma} \eta(e) \geq B_{\alpha} \rho\|x\|_{1}\right) \\
\leq \sum_{\gamma,|\gamma| \leq \rho\|x\|_{1}} \mathbb{P}_{p}(0 \leftrightarrow x ; \gamma(0, x)=\gamma) S_{\nu}\left(\sum_{e \in \gamma} \eta(e) \geq B_{\alpha}|\gamma|\right) \\
\leq \sum_{\gamma,|\gamma| \leq \rho\|x\|_{1}} \mathbb{P}_{p}(0 \leftrightarrow x ; \gamma(0, x)=\gamma) \frac{A_{\alpha}}{|\gamma|^{\alpha}} \text { with Assumption }\left(H_{\alpha}\right) \\
\leq \frac{A_{\alpha} \mathbb{P}_{p}(0 \leftrightarrow x)}{\|x\|_{1}^{\alpha}} \text { as }|\gamma(0, x)| \geq\|x\|_{1} .
\end{aligned}
$$

This gives the announced estimate.

Antal and Pisztora's result (1) gives a control of the chemical distance $D(0, x)$ for large $x$. The next lemma, which is a very rough bound, will nevertheless be sufficient to deal with small $x$ :

Lemma 2.2. There exists two positive constants $C_{\rho}, D_{\rho}$, depending only on the dimension $d$, such that for every $r \geq 1$, for every $x \in \mathbb{Z}^{d}$ such that $\|x\|_{1} \leq r$,

$$
\mathbb{P}_{p}\left(0 \leftrightarrow x ; D(0, x) \geq(3 r)^{d}\right) \leq C_{\rho} \exp \left(-D_{\rho} r\right)
$$

Proof. Take $\rho, A_{\rho}, B_{\rho}$ as given by (1). Assume without loss of generality that $r \geq \max \left\{2,\left(\frac{10 \rho}{3^{d}}\right)^{\frac{1}{d-1}}\right\}$ and take $x \in \mathbb{Z}^{d}$ such that $\|x\|_{1} \leq r$.

We denote by $B(r)$ the cube $[-5 r / 4,5 r / 4]^{d} \cap \mathbb{Z}^{d}$. As the segment $[a, b]$ contains at most $b-a+1$ integer points, one has $|B(r)| \leq(1+5 r / 2)^{d}$, and then, as $r \geq 2,|B(r)|<(3 r)^{d}$. Denote by $\partial B(r)$ the external boundary of $B(r)$, defined as the set of vertices in $\mathbb{Z}^{d} \backslash B(r)$ that admit a neighbour in $B(r)$. Note that $x \in B(r)$ and that if $y \in \partial B(r)$, then $\|x-y\|_{1} \geq r / 4$.

Assume that $D(0, x)>(3 r)^{d}$. Then $D(0, x)>|B(r)|$, and thus $\gamma(0, x)$ must visit at least one vertex in $\partial B(r)$ :

$$
\begin{aligned}
\mathbb{P}\left(0 \leftrightarrow x, D(0, x) \geq(3 r)^{d}\right) & \leq \mathbb{P}\left(\exists y \in \partial B(r) \text { such that } 0 \leftrightarrow y, y \leftrightarrow x, D(0, y) \geq \frac{(3 r)^{d}}{2} \text { or } D(x, y) \geq \frac{(3 r)^{d}}{2}\right) \\
& \leq \sum_{y \in \partial B(r)} \mathbb{P}\left(0 \leftrightarrow y, D(0, y) \geq \frac{(3 r)^{d}}{2}\right)+\mathbb{P}\left(x \leftrightarrow y, D(x, y) \geq \frac{(3 r)^{d}}{2}\right) .
\end{aligned}
$$

But, by the choice we made for $r, \frac{(3 r)^{d}}{2} \geq 5 \rho r \geq \rho \max \left\{\|y\|_{1},\|x-y\|_{1}\right\}$, and thus

$$
\mathbb{P}\left(0 \leftrightarrow x, D(0, x) \geq(3 r)^{d}\right) \leq \sum_{y \in \partial B(r)} \mathbb{P}\left(0 \leftrightarrow y, D(0, y) \geq \rho\|y\|_{1}\right)+\mathbb{P}\left(x \leftrightarrow y, D(x, y) \geq \rho\|x-y\|_{1}\right)
$$


But $\min \left\{\|y\|_{1},\|x-y\|_{1}\right\} \geq \frac{r}{4}$ and using (1) we get:

$$
\begin{aligned}
\mathbb{P}\left(0 \leftrightarrow x, D(0, x) \geq(3 r)^{d}\right) & \leq 2 \sum_{y \in \partial B(r)} A_{\rho} \exp \left(-\frac{B_{\rho} r}{4}\right) \\
& \leq 2(2 d)\left(3+\frac{5 r}{2}\right)^{d-1} A_{\rho} \exp \left(-\frac{B_{\rho} r}{4}\right) .
\end{aligned}
$$

This ends the proof of the lemma.

We can now obtain a similar control for the travel times:

Lemma 2.3. If $\left(H_{\alpha}\right)$ holds for some $\alpha>0$, then there exist positive constants $a_{\alpha}$ and $D_{\alpha}$, depending only on the dimension $d$ of the grid, such that for every $r \geq 1$, for every $x \in \mathbb{Z}^{d}$ satisfying $\|x\|_{1} \leq r$,

$$
\mathbb{P}\left(0 \leftrightarrow x, d(0, x) \geq\left(a_{\alpha} r\right)^{d}\right) \leq \frac{D_{\alpha}}{r^{\nu}},
$$

with $\nu=\alpha-d+1$.

Proof. Denote as previously by $B(r)$ the cube $[-5 r / 4,5 r / 4]^{d} \cap \mathbb{Z}^{d}$, and by $\partial B(r)$ the set of vertices in $\mathbb{Z}^{d} \backslash B(r)$ that admit a neighbour inside $B(r)$. We have $|B(r)| \leq(1+5 r / 2)^{d}$. Denote by $n_{e}(r)$ the number of edges that have each of their extremities in $B(r)$. Then

$$
d(5 r / 2-2)^{d} \leq n_{e}(r) \leq d(5 r / 2)^{d} .
$$

Now, let $A_{\alpha}$ and $B_{\alpha}$ be the two constants given by Assumption $\left(H_{\alpha}\right)$ and choose $a_{\alpha}$ such that:

$$
\forall r \text { large enough, }\left(a_{\alpha} r\right)^{d}>B_{\alpha} d\left(\frac{5 r}{2}\right)^{d} .
$$

Take now $x \in \mathbb{Z}^{d}$ such that $\|x\|_{1} \leq r$. Note that $x \in B(r)$. Now,

$$
\begin{aligned}
\mathbb{P}\left(0 \leftrightarrow x, d(0, x) \geq\left(a_{\alpha} r\right)^{d}\right)= & \mathbb{P}\left(0 \leftrightarrow x, d(0, x) \geq\left(a_{\alpha} r\right)^{d} \text { and } \sum_{e \in B(r)} \eta_{e}<B_{\alpha} d\left(\frac{5 r}{2}\right)^{d}\right) \\
& +\mathbb{P}\left(0 \leftrightarrow x, d(0, x) \geq\left(a_{\alpha} r\right)^{d} \text { and } \sum_{e \in B(r)} \eta_{e} \geq B_{\alpha} d\left(\frac{5 r}{2}\right)^{d}\right) \\
\leq & \mathbb{P}\left(0 \leftrightarrow x, d(0, x) \geq\left(a_{\alpha} r\right)^{d} \text { and } \sum_{e \in B(r)} \eta_{e}<B_{\alpha} d\left(\frac{5 r}{2}\right)^{d}\right) \\
& +\mathbb{P}\left(\sum_{e \in B(r)} \eta_{e} \geq B_{\alpha} n_{e}(r)\right) .
\end{aligned}
$$

By Assumption $\left(H_{\alpha}\right)$, the last term is smaller than $\frac{A_{\alpha}}{n_{e}(r)^{\alpha}} \leq \frac{A_{\alpha}}{d^{\alpha}(5 r / 2-2)^{d \alpha}}$.

If

$$
\left\{0 \leftrightarrow x, d(0, x) \geq\left(a_{\alpha} r\right)^{d} \text { and } \sum_{e \in B(r)} \eta_{e}<B_{\alpha} d\left(\frac{5 r}{2}\right)^{d}\right\}
$$


is satisfied, then $\gamma(0, x)$ necessarily goes out of $B(0, r)$. Note moreover that for $r$ large enough and $y \in \partial B(r)$, one has

$$
\begin{aligned}
B_{\alpha} \rho\|y\|_{1} \leq B_{\alpha} \rho(5 r / 4+1) \leq\left(a_{\alpha} r\right)^{d} / 2 \quad & \text { and } \quad\|y\|_{1} \geq r / 4 \\
B_{\alpha} \rho\|x-y\|_{1} \leq B_{\alpha} \rho(5 r / 4+1) \leq\left(a_{\alpha} r\right)^{d} / 2 & \text { and } \quad\|y-x\|_{1} \geq r / 4 .
\end{aligned}
$$

When $r$ is large enough, the first term is thus controlled by

$$
\begin{aligned}
\mathbb{P}(0 & \left.\leftrightarrow x, d(0, x) \geq\left(a_{\alpha} r\right)^{d} \text { and } \sum_{e \in B(r)} \eta_{e}<B_{\alpha} d\left(\frac{5 r}{2}\right)^{d}\right) \\
& \leq \mathbb{P}\left(\exists y \in \partial B(r) \text { such that } 0 \leftrightarrow y, y \leftrightarrow x, d(0, y) \geq \frac{\left(a_{\alpha} r\right)^{d}}{2} \text { or } d(x, y) \geq \frac{\left(a_{\alpha} r\right)^{d}}{2}\right) \\
& \leq \sum_{y \in \partial B(r)} \mathbb{P}\left(0 \leftrightarrow y, d(0, y) \geq \frac{\left(a_{\alpha} r\right)^{d}}{2}\right)+\mathbb{P}\left(x \leftrightarrow y, d(x, y) \geq \frac{\left(a_{\alpha} r\right)^{d}}{2}\right) \\
& \leq \sum_{y \in \partial B(r)} \mathbb{P}\left(0 \leftrightarrow y, d(0, y) \geq B_{\alpha} \rho\|y\|_{1}\right)+\mathbb{P}\left(x \leftrightarrow y, d(x, y) \geq B_{\alpha} \rho\|x-y\|_{1}\right) \\
& \leq \sum_{y \in \partial B(r)} C_{\alpha}\left(\frac{1}{\|y\|_{1}^{\alpha}}+\frac{1}{\|x-y\|_{1}^{\alpha}}\right) \quad \text { with Lemma } 2.1 \\
& \leq 4 d\left(\frac{5 r}{2}+3\right)^{d-1} C_{\alpha}\left(\frac{4}{r}\right)^{\alpha} .
\end{aligned}
$$

To conclude, note that as $d \geq 2$ and $\alpha>0$, we always have $d \alpha \geq \alpha-d+1$, thus this last term is always the main one.

We can now give an uniform bound for the expectation of the chemical distance between the origin and $T_{1, u} u=T_{u} u$ :

Lemma 2.4. There exist a function $\psi: \mathbb{Z}^{d} \rightarrow \mathbb{R}^{+}$satisfying $\lim _{\|u\|_{1} \rightarrow+\infty} \psi(u)=0$, and such that

$$
\forall u \in \mathbb{Z}^{d}, \quad \mathbb{E}_{\overline{\mathbb{P}}_{p}}\left(D\left(0, T_{u} u\right)\right) \leq \frac{\|u\|_{1}}{\mathbb{P}_{p}(0 \leftrightarrow \infty)}(\rho+\psi(u))
$$

The constant $\rho$ is the one given by (1).

Proof. Recall the following identity:

$$
\mathbb{E}_{\overline{\mathbb{P}}_{p}} \frac{D\left(0, T_{u} u\right)}{\|u\|}=\int_{0}^{+\infty} \overline{\mathbb{P}}_{p}\left(D\left(0, T_{u} u\right) \geq h\|u\|_{1}\right) \mathrm{d} h,
$$


and cut $\mathbb{E}_{\overline{\mathbb{P}}}\left(D\left(0, T_{u} u\right)\right)$ in three terms, according to the values of $T_{u}$. Let us estimate first the contribution for $T_{u}$ small:

$$
\begin{aligned}
S_{1}(h, u) & =\overline{\mathbb{P}}_{p}\left(D\left(0, T_{u} u\right) \geq h\|u\|_{1}, T_{u} \leq \frac{\left(h\|u\|_{1}\right)^{1 / d}}{3\|u\|_{1}}\right) \\
& =\frac{1}{\mathbb{P}_{p}(0 \leftrightarrow \infty)} \mathbb{P}_{p}\left(D\left(0, T_{u} u\right) \geq h\|u\|_{1}, T_{u} \leq \frac{\left(h\|u\|_{1}\right)^{1 / d}}{3\|u\|_{1}}\right) \\
& =\frac{1}{\mathbb{P}_{p}(0 \leftrightarrow \infty)} \sum_{1 \leq k \leq \frac{\left(h\|u\|_{1}\right)^{1 / d}}{3\|u\|_{1}}} \mathbb{P}_{p}\left(0 \leftrightarrow k u, D(0, k u) \geq h\|u\|_{1}, T_{u}=k\right) \\
& \leq \frac{1}{\mathbb{P}_{p}(0 \leftrightarrow \infty)} \sum_{1 \leq k \leq \frac{\left(h\|u\|_{1}\right)^{1 / d}}{3\|u\|_{1}}} \mathbb{P}_{p}\left(0 \leftrightarrow k u, D(0, k u) \geq h\|u\|_{1}\right) .
\end{aligned}
$$

But as $k \leq \frac{\left(h\|u\|_{1}\right)^{1 / d}}{3\|u\|_{1}}$, we have $h\|u\|_{1} \geq\left(3 k\|u\|_{1}\right)^{d}$ and thus, using Lemma 2.2 with $r=\frac{\left(h\|u\|_{1}\right)^{1 / d}}{3}$ and $x=k u$, we get

$$
\begin{aligned}
S_{1}(h, u) & \leq \frac{1}{\mathbb{P}_{p}(0 \leftrightarrow \infty)} \sum_{1 \leq k \leq \frac{\left(h\|u\|_{1}\right)^{1 / d}}{3\|u\|_{1}}} \mathbb{P}_{p}\left(0 \leftrightarrow k u, D(0, k u) \geq(3 r)^{d}\right) \\
& \leq \frac{1}{\mathbb{P}_{p}(0 \leftrightarrow \infty)} \frac{\left(h\|u\|_{1}\right)^{1 / d}}{3\|u\|_{1}} C_{\rho} \exp \left(-D_{\rho} \frac{\left(h\|u\|_{1}\right)^{1 / d}}{3}\right) .
\end{aligned}
$$

Now, $\int_{\mathbb{R}^{+}} S_{1}(h, u) \mathrm{d} h$ is finite:

$$
\begin{aligned}
\int_{0}^{\infty} S_{1}(h, u) \mathrm{d} h & \leq \frac{1}{\mathbb{P}_{p}(0 \leftrightarrow \infty)} \int_{0}^{\infty} \frac{\left(h\|u\|_{1}\right)^{1 / d}}{3\|u\|_{1}} C_{\rho} \exp \left(-D_{\rho} \frac{\left(h\|u\|_{1}\right)^{1 / d}}{3}\right) \mathrm{d} h \\
& \leq \frac{1}{\mathbb{P}_{p}(0 \leftrightarrow \infty)} \frac{C_{\rho}}{3\|u\|_{1}^{2}} \int_{0}^{\infty} x^{1 / d} \exp \left(-D_{\rho} \frac{x^{1 / d}}{3}\right) \mathrm{d} x .
\end{aligned}
$$

Thus there exists a constant $C_{1}$ such that for every $u \in \mathbb{Z}^{d}$ :

$$
\int_{0}^{\infty} S_{1}(h, u) \mathrm{d} h=\int_{0}^{\infty} \overline{\mathbb{P}}_{p}\left(D\left(0, T_{u} u\right) \geq h\|u\|_{1}, T_{u} \leq \frac{\left(h\|u\|_{1}\right)^{1 / d}}{3\|u\|_{1}}\right) \mathrm{d} h \leq \frac{C_{1}}{\|u\|_{1}^{2}}
$$


Let us now estimate the contribution for $T_{u}$ medium:

$$
\begin{aligned}
S_{2}(h, u) & =\overline{\mathbb{P}}_{p}\left(D\left(0, T_{u} u\right) \geq h\|u\|_{1}, \frac{\left(h\|u\|_{1}\right)^{1 / d}}{3\|u\|_{1}}<T_{u} \leq \frac{h}{\rho}\right) \\
& =\frac{1}{\mathbb{P}_{p}(0 \leftrightarrow \infty)} \mathbb{P}_{p}\left(0 \leftrightarrow \infty, D\left(0, T_{u} u\right) \geq h\|u\|_{1}, \frac{\left(h\|u\|_{1}\right)^{1 / d}}{3\|u\|_{1}}<T_{u} \leq \frac{h}{\rho}\right) \\
& =\frac{1}{\mathbb{P}_{p}(0 \leftrightarrow \infty)} \sum_{\frac{\left(h\|u\|_{1}\right)^{1 / d}}{3\|u\|_{1}}<k \leq \frac{h}{\rho}} \mathbb{P}_{p}\left(0 \leftrightarrow \infty, D\left(0, T_{u} u\right) \geq h\|u\|_{1}, T_{u}=k\right) \\
& \leq \frac{1}{\mathbb{P}_{p}(0 \leftrightarrow \infty)} \sum_{\frac{\left(h\|u\|_{1}\right)^{1 / d}}{3\|u\|_{1}}<k \leq \frac{h}{\rho}} \mathbb{P}_{p}\left(0 \leftrightarrow k u, D(0, k u) \geq h\|u\|_{1}\right) \\
& \leq \frac{1}{\mathbb{P}_{p}(0 \leftrightarrow \infty)} \sum_{\frac{\left(h\|u\|_{1}\right)^{1 / d}}{3\|u\|_{1}}<k \leq \frac{h}{\rho}} \mathbb{P}_{p}\left(0 \leftrightarrow k u, D(0, k u) \geq \rho k\|u\|_{1}\right) \\
& \leq \frac{1}{\mathbb{P}_{p}(0 \leftrightarrow \infty)} \sum_{\frac{\left(h\|u\|_{1}\right)^{1 / d}}{3\|u\|_{1}}<k \leq \frac{h}{\rho}} A_{\rho} \exp \left(-B_{\rho} k\|u\|_{1}\right) \\
& \leq \frac{1}{\mathbb{P}_{p}(0 \leftrightarrow \infty)} \frac{h}{\rho} A_{\rho} \exp \left(-B_{\rho} \frac{\left(h\|u\|_{1}\right)^{1 / d}}{3}\right) .
\end{aligned}
$$

Thus, there exists a constant $C_{2}$, independent from $u$, such that

$$
\begin{aligned}
\int_{0}^{\infty} S_{2}(h, u) \mathrm{d} h & \leq \frac{1}{\mathbb{P}_{p}(0 \leftrightarrow \infty)} \int_{0}^{\infty} \frac{h}{\rho} A_{\rho} \exp \left(-B_{\rho} \frac{\left(h\|u\|_{1}\right)^{1 / d}}{3}\right) \mathrm{d} h \\
& \leq \frac{1}{\mathbb{P}_{p}(0 \leftrightarrow \infty)} \frac{A_{\rho}}{\rho\|u\|_{1}^{2}} \int_{0}^{\infty} x \exp \left(-B_{\rho} \frac{x^{1 / d}}{3}\right) \mathrm{d} x \\
& \leq \frac{C_{2}}{\|u\|_{1}^{2}} .
\end{aligned}
$$

Finally, for $T_{u}$ large:

$$
\begin{aligned}
S_{3}(h, u) & =\overline{\mathbb{P}}_{p}\left(D\left(0, T_{u} u\right) \geq h\|u\|_{1}, T_{u}>\frac{h}{\rho}\right) \\
& \leq \overline{\mathbb{P}}_{p}\left(T_{u}>\frac{h}{\rho}\right)
\end{aligned}
$$

and

$$
\begin{aligned}
\int_{0}^{+\infty} S_{3}(h, u) \mathrm{d} h & \leq \int_{0}^{+\infty} \overline{\mathbb{P}}_{p}\left(T_{u}>\frac{h}{\rho}\right) \mathrm{d} h \\
& \leq \rho \mathbb{E}_{\overline{\mathbb{P}}_{p}} T_{u} \quad \text { with Lemma } 1.3 \\
& \leq \frac{\rho}{\mathbb{P}_{p}(0 \leftrightarrow \infty)} .
\end{aligned}
$$

Putting these three pieces together, we get the announced result. 
We finally prove the integrability of the travel time $d\left(0, T_{u} u\right)$ :

Corollary 2.5. If $\left(H_{\text {int }}\right)$ holds, then for every $u \in \mathbb{Z}^{d} \backslash\{0\}$,

$$
\mathbb{E}_{\overline{\mathbb{P}}}\left(d\left(0, T_{u} u\right)\right) \leq m \mathbb{E}_{\overline{\mathbb{P}}_{p}}\left(D\left(0, T_{u} u\right)\right)<\infty .
$$

Proof. This is due to a simple comparison argument:

$$
\begin{aligned}
\mathbb{E}_{\overline{\mathbb{P}}}\left(d\left(0, T_{u} u\right)\right) & =\sum_{k=0}^{+\infty} \mathbb{E}_{\overline{\mathbb{P}}}\left(d(0, k u) \mathbb{1}_{\left\{T_{u}=k\right\}}\right) \\
& \leq \sum_{k=0}^{+\infty} \mathbb{E}_{\overline{\mathbb{P}}}\left(\mathbb{1}_{\left\{T_{u}=k\right\}} \sum_{e \in \gamma(0, k u)} \eta(e)\right) \\
& \leq \sum_{k=0}^{+\infty} \mathbb{E}_{\overline{\mathbb{P}}}\left(\mathbb{1}_{\left\{T_{u}=k\right\}} \mathbb{E}_{\overline{\mathbb{P}}}\left(\sum_{e \in \gamma(0, k u)} \eta(e) \mid \omega\right)\right) \\
& \leq \sum_{k=0}^{+\infty} \mathbb{E}_{\overline{\mathbb{P}}_{p}}\left(\mathbb{1}_{\left\{T_{u}=k\right\}} m D(0, k u)\right) \\
& \leq m \mathbb{E}_{\overline{\mathbb{P}}_{p}}\left(D\left(0, T_{u} u\right)\right) .
\end{aligned}
$$

Lemma 2.4 gives then the integrability of $d\left(0, T_{u} u\right)$.

\section{Existence of Directional time constants And Related Properties}

The next lemma is the analogous of the first step in the classical study of first-passage percolation, that is the proof of the existence of an asymptotic time constant in a given direction.

Unsurprisingly, the fundamental tool is here again Kingman's sub-additive ergodic theorem. The main difference is that it is applied to an induced dynamical system, and not directly. It is the reason why checking the integrability (Cor. 2.5) was more intricate than in the classical case.

Lemma 3.1. For $n \geq 0$ and $u \in \mathbb{Z}^{d} \backslash\{0\}$, we define the travel time $f_{n, u}$ between the origin and the $n$-th intersection of the infinite cluster with $\mathbb{Z}_{+}^{*} u$ :

$$
f_{n, u}(\omega, \eta)=d\left(0,\left(T_{n, u}(\omega) u\right)\right)(\omega, \eta) .
$$

Then there exists a constant $f_{u} \geq 0$ such that

$$
\frac{f_{n, u}}{n} \rightarrow f_{u} \quad \overline{\mathbb{P}} \text { a.s. }
$$

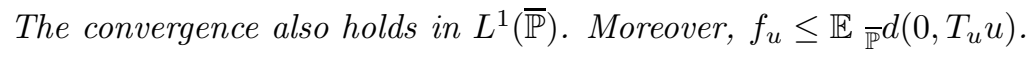

Proof. For the convenience of the reader, we now fix $u \in \mathbb{Z}^{d} \backslash\{0\}$ and write $f_{n}$ (resp. $f, T_{n}, T$ ) instead of $f_{n, u}$ (resp. $f_{u}, T_{n, u}, T_{u}$ ).

Since the travel time is a sub-additive function, we have:

$$
\begin{aligned}
f_{n+k} & =d\left(0, T_{n+k} u\right) \\
& \leq d\left(0, T_{n} u\right)+d\left(T_{n} u, T_{n+k} u\right) \\
& =d\left(0, T_{n} u\right)+d\left(0, T_{k} u\right) \circ \Theta_{u}^{n} \\
& =f_{n}+f_{k} \circ \Theta_{u}^{n} .
\end{aligned}
$$


Moreover, the $f_{n}$ are non-negative and, thanks to Lemma 1.3, $\overline{\mathbb{P}}$ is invariant under $\Theta_{u}$. Then, as $f_{1}$ is integrable thanks to Lemma 2.5, Kingman's sub-additive ergodic theorem says there exists a $\Theta_{u}$-invariant function $f$ such that

$$
\frac{f_{n}}{n} \rightarrow f \overline{\mathbb{P}} \text { a.s. and in } L^{1}(\overline{\mathbb{P}}) \text {. }
$$

Since $\Theta_{u}$ is ergodic for $\overline{\mathbb{P}}$, every $\Theta_{u}$-invariant function is $\overline{\mathbb{P}}$ almost surely constant, so $f$ is constant. Moreover, since every subadditive real sequence $\left(a_{n}\right)_{n \in \mathbb{Z}_{+}}$converges to $\inf \left(a_{n} / n\right)$,

$$
\mathbb{E}_{\mathbb{P}} f=\inf _{n \geq 0} \frac{\mathbb{E}}{n} f_{n} \leq \mathbb{E}_{\bar{P}} f_{1}=\mathbb{E}_{\bar{P}} d\left(0, T_{u}\right) .
$$

We can now define a function $\mu$ with the same properties as the time constant of first-passage percolation.

Theorem 3.2 (Existence of directional time constants). Assume that $\left(H_{\alpha}\right)$ holds for some $\alpha>1$. For each $u \in \mathbb{Z}^{d} \backslash\{0\}$, we set $\mu(u)=\mathbb{P}_{p}(0 \leftrightarrow \infty) f_{u}$, where $f_{u}$ is given by Lemma 3.1, and $\mu(0)=0$. Then

$$
\lim _{n \rightarrow \infty} \frac{d\left(0, T_{n, u} u\right)}{T_{n, u}}=\mu(u) \quad \overline{\mathbb{P}} \text { a.s. }
$$

Moreover, this function $\mu: \mathbb{Z}^{d} \rightarrow[0, \infty)$ has the following properties:

(1) $\mu$ is symmetric: for any vector $u \in \mathbb{Z}^{d}: \mu(-u)=\mu(u)$.

If, moreover, $S_{\nu}$ is a product measure, then $\mu$ is invariant under permutations or reflections of the coordinates in $\mathbb{Z}^{d}$.

(2) $\mu$ is homogeneous: for any integer $k \in \mathbb{Z}$ and any vector $u \in \mathbb{Z}^{d}$,

$$
\mu(k u)=|k| \mu(u)
$$

(3) $\mu$ is locally bounded: for any vector $u \in \mathbb{Z}^{d}$,

$$
\mu(u) \leq \rho m\|u\|_{1} \quad \text { and } \mu(u) \leq \mu_{*}\|u\|_{1},
$$

with $\mu_{*}=\sup _{1<i<d} \mu\left(e_{i}\right)$.

(4) $\mu$ is sub-additive, in the following sense: for any vectors $u$ and $v$ in $\mathbb{Z}^{d}$,

$$
\mu(u+v) \leq \mu(u)+\mu(v) .
$$

(5) $\mu$ is continuous: for any vectors $u$ and $v$ in $\mathbb{Z}^{d}$,

$$
|\mu(u)-\mu(v)| \leq \mu_{*}\|u-v\|_{1} .
$$

We delay the proof of this theorem to make some remarks and comments.

Remarks. 1. Let us now discuss the meaning of the inequality $\mu(u) \leq \rho m\|u\|_{1}$. In classical first-passage percolation, this would correspond to the inequality $\mu(u) \leq m\|u\|_{1}$. In our case, the usual $\|\cdot\|_{1}$ distance is replaced by the random chemical distance $D$; but the Corollary 1.3 of Antal and Pisztora [2] gives a bound for the asymptotic ratio between the chemical distance and the $\|\cdot\|_{1}$ distance on $\mathbb{Z}^{d}$ :

$$
\varlimsup_{\|y\|_{1} \rightarrow+\infty} \frac{D(0, y)}{\|y\|_{1}} \mathbb{1}_{\{0 \leftrightarrow y\}} \leq \rho(p, d) \mathbb{P}_{p} \text { a.s. }
$$

Thus the additional factor $\rho$ corresponds to this upper bound for the asymptotic ratio between the chemical distance and the $\|\cdot\|_{1}$ distance on $\mathbb{Z}^{d}$. 
2. In the special case $S_{\nu}=\left(\delta_{1}\right)^{\otimes \mathbb{E}^{d}}$, the travel times coincide with the chemical distances. As a direct corollary of Theorem 3.2, we obtain the existence of asymptotic directional time constants for the chemical distance:

Corollary 3.3 (directional time constants for chemical distance in supercritical Bernoulli percolation). Assume that $d \geq 2$ and that $p>p_{c}(d)$, and consider the Bernoulli percolation model with parameter $p$ on the edges of $\mathbb{Z}^{d}$. Then for each $y \in \mathbb{Z}^{d} \backslash\{0\}$, there exists a constant $\mu(y)$ such that

$$
\lim _{\substack{n \rightarrow+\infty \\ 0 \leftrightarrow n y}} \frac{D(0, n y)}{n}=\mu(y) \mathbb{P}_{p} \text { a.s. }
$$

Note that this specifies the equation (2) of Antal and Pisztora.

Proof of Theorem 3.2.

Step 1. The convergence result is clear since $T_{n, u} / n \rightarrow 1 / \mathbb{P}_{p}\left(0 \in C_{\infty}\right)$ and $f_{n, u} / n \rightarrow f_{u} \geq 0$. Consequently, we obtain the identity $\mu(u)=\mathbb{P}_{p}(0 \leftrightarrow \infty) f_{u}$.

Step 2. It is easy to see that $\frac{d(0, n u)}{n}$ weakly converges to

$$
\mathbb{P}_{p}(0 \leftrightarrow \infty) \delta_{f_{u}}+\left(1-\mathbb{P}_{p}(0 \leftrightarrow \infty)\right) \delta_{+\infty} .
$$

By translation invariance $d(0, n u)=d(n u, 0)$ has the same law that $d(0,-n u)$. It follows that $f_{u}=f_{-u}$, and then that $\mu(u)=\mu(-u)$.

When $S_{\nu}$ is a product measure, Property (1) is obvious since the whole model is invariant under permutations or reflections of the coordinates in $\mathbb{Z}^{d}$.

Step 3. Let us prove now that for any integer $k \in \mathbb{Z} \backslash\{0\}$ and any non null vector $u \in \mathbb{Z}^{d}, \mu(k u)=|k| \mu(u)$. Note that

$$
\frac{f_{n, k u}}{n}=|k| \frac{f_{n, k u}}{n|k|} ;
$$

but $\left(f_{n, k u} / n|k|\right)_{n \geq 1}$ is a subsequence of $\left(f_{n, \varepsilon u} / n\right)_{n \geq 1}$ with $\varepsilon=k /|k|$. Thus

$$
\frac{\mu(k u)}{\mathbb{P}_{p}(0 \leftrightarrow \infty)}=\lim _{n \rightarrow+\infty} \frac{f_{n, k u}}{n}=|k| \lim _{n \rightarrow+\infty} \frac{f_{n, \varepsilon u}}{n}=|k| \frac{\mu(\varepsilon u)}{\mathbb{P}_{p}(0 \leftrightarrow \infty)}=|k| \frac{\mu(u)}{\mathbb{P}_{p}(0 \leftrightarrow \infty)},
$$

by steps 1 and 2 .

Step 4. By the previous step $\mu(u)=\frac{1}{n} \mu(n u)$. Then

$$
\begin{aligned}
\mu(u) & =\mathbb{P}(0 \leftrightarrow \infty) \frac{f_{n u}}{n} \\
& \leq \frac{\mathbb{E}_{\overline{\mathbb{P}}} d\left(0, T_{n u} n u\right)}{n} \mathbb{P}(0 \leftrightarrow \infty) \\
& \leq \frac{m \mathbb{E} \overline{\mathbb{P}} D\left(0, T_{n u} n u\right)}{n} \mathbb{P}(0 \leftrightarrow \infty) \text { by Corollary } 2.5 \\
& \leq \frac{m(\rho+\psi(n u))\|n u\|_{1}}{n} \text { by Lemma } 2.4 \\
& \leq m(\rho+\psi(n u))\|u\|_{1} .
\end{aligned}
$$


Since $\lim _{n \rightarrow+\infty} \psi(n u)=0$, it follows that $\mu(u) \leq m \rho\|u\|_{1}$. This proves the first part of (3).

Step 5. Let us now prove the sub-additive property of $\mu$. The main difficulty here, compared to the classical proof in standard first-passage percolation (see Kesten [13]) is that a realization of the environment is not translation invariant.

We thus use Cesaro means and decorrelation properties of the classical Bernoulli percolation to recover the sub-additive property of $\mu$.

Lemma 3.4. Let $x$ and $y$ be two linearly independent vectors in $\mathbb{Z}^{d}$. Then

$$
\frac{1}{n} \sum_{k=1}^{n} \mathbb{1}_{\{k x \leftrightarrow \infty\}} \mathbb{1}_{\{k y \leftrightarrow \infty\}} \rightarrow \mathbb{P}_{p}(0 \leftrightarrow \infty)^{2} \text { almost surely under } \mathbb{P}_{p}
$$

Proof. Let $\alpha=\frac{1}{3} \min \left(\|x\|_{1},\|y\|_{1},\|x-y\|_{1}\right)$ and define $Z_{k}=\mathbb{1}_{\{\alpha k \leq|C(k x)|\}} \mathbb{1}_{\{\alpha k \leq|C(k y)|\}}$.

$$
\begin{aligned}
\mathbb{P}_{p}\left(Z_{k} \neq \mathbb{1}_{\{k x \leftrightarrow \infty\}} \mathbb{1}_{\{k y \leftrightarrow \infty\}}\right) & \leq \mathbb{P}_{p}(\alpha k \leq|C(k x)|<+\infty)+\mathbb{P}_{p}(\alpha k \leq|C(k y)|<+\infty) \\
& \leq 2 \mathbb{P}_{p}(\alpha k \leq|C(0)|<+\infty) .
\end{aligned}
$$

Since $p>p_{c}$, a result of Kesten and Zhang in [14] ensures that $|C(0)| \mathbb{1}_{\{|C(0)|<+\infty\}}$ is integrable, and we get

$$
\sum_{k \geq 1} \mathbb{P}_{p}\left(Z_{k} \neq \mathbb{1}_{\{k x \leftrightarrow \infty\}} \mathbb{1}_{\{k y \leftrightarrow \infty\}}\right)<+\infty .
$$

Hence, Borel-Cantelli lemma ensures that, $\mathbb{P}_{p}$ almost surely, $Z_{k}$ coincides with $\mathbb{1}_{\{k x \leftrightarrow \infty\}} \mathbb{1}_{\{k y \leftrightarrow \infty\}}$ for every $k$ large enough. It follows that $\frac{1}{n} \sum_{k=1}^{n} \mathbb{1}_{\{k x \leftrightarrow \infty\}} \mathbb{1}_{\{k y \leftrightarrow \infty\}}$ has the same limit that $\frac{1}{n} \sum_{k=1}^{n} Z_{k}$. But $\left(Z_{k}\right)_{k \geq 1}$ is a sequence of independent uniformly bounded random variables. Then

$$
\frac{1}{n} \sum_{k=1}^{n}\left(Z_{k}-\mathbb{E}_{\mathbb{P}_{p}} Z_{k}\right) \rightarrow 0 \mathbb{P}_{p} \text { a.s. }
$$

Since $\alpha=\frac{1}{3} \min \left(\|x\|_{1},\|y\|_{1},\|x-y\|_{1}\right)$,

$$
\mathbb{E}_{\mathbb{P}_{p}} Z_{k}=\mathbb{E}_{\mathbb{P}_{p}} \mathbb{1}_{\{\alpha k \leq|C(k x)|\}} \mathbb{E}_{\mathbb{P}_{p}} \mathbb{1}_{\{\alpha k \leq|C(k y)|\}}=\mathbb{P}_{p}(\alpha k \leq C(0))^{2} \rightarrow \mathbb{P}_{p}(0 \leftrightarrow \infty)^{2}
$$

and the result follows from Cesaro's theorem.

Lemma 3.5. Assume that there exists $\alpha>1$ such that $\left(H_{\alpha}\right)$ is fulfilled with two positive constants $A_{\alpha}$ and $B_{\alpha}$, and let $\rho$ be the constant that appears in (1). Let $x$ and $y$ be two linearly independent vectors in $\mathbb{Z}^{d}$, and for $k \in \mathbb{Z}_{+}^{*}$, denote by $A_{k}$ the following event:

$$
A_{k}=\left\{d(0, k x) \leq B_{\alpha} \rho k\|x\|_{1}, d(0, k(x+y)) \leq B_{\alpha} \rho k\|x+y\|_{1}, d(k x, k(x+y)) \leq B_{\alpha} \rho k\|y\|_{1}\right\} .
$$

Consider the following sequence:

$$
u_{n}=\frac{1}{n} \sum_{k=1}^{n} \frac{d(0, k x)}{k} \mathbb{1}_{A_{k}} \mathbb{1}_{\{0 \leftrightarrow \infty\}} \mathbb{1}_{\{k x \leftrightarrow \infty\}} \mathbb{1}_{\{k(x+y) \leftrightarrow \infty\}} .
$$

Then, $\left(u_{n}\right)_{n \geq 1} \mathbb{P}$ almost surely converges to $\mu(x) \mathbb{1}_{\{0 \leftrightarrow \infty\}} \mathbb{P}_{p}(0 \leftrightarrow \infty)^{2}$ and moreover, $\left(\mathbb{E}_{\mathbb{P}}\left(u_{n}\right)\right)_{n \geq 1}$ converges to $\mu(x) \mathbb{P}_{p}(0 \leftrightarrow \infty)^{3}$. 
Proof. Note first that

$$
\begin{aligned}
& \mathbb{P}_{p}\left(\mathbb{1}_{A_{k}} \mathbb{1}_{\{0 \leftrightarrow \infty\}} \mathbb{1}_{\{k x \leftrightarrow \infty\}} \mathbb{1}_{\{k(x+y) \leftrightarrow \infty\}} \neq \mathbb{1}_{\{0 \leftrightarrow \infty\}} \mathbb{1}_{\{k x \leftrightarrow \infty\}} \mathbb{1}_{\{k(x+y) \leftrightarrow \infty\}}\right) \\
&= \mathbb{E}_{\mathbb{P}_{p}}\left(\mathbb{1}_{\{0 \leftrightarrow \infty\}} \mathbb{1}_{\{k x \leftrightarrow \infty\}} \mathbb{1}_{\{k(x+y) \leftrightarrow \infty\}}\left(1-\mathbb{1}_{A_{k}}\right)\right) \\
& \leq \mathbb{E}_{\mathbb{P}_{p}}\left[\mathbb{1}_{\{0 \leftrightarrow \infty\}} \mathbb{1}_{\{k x \leftrightarrow \infty\}} \mathbb{1}_{\{k(x+y) \leftrightarrow \infty\}}\right. \\
&\left.\times\left(\mathbb{1}_{\left\{d(0, k x)>B_{\alpha} \rho k\|x\|_{1}\right\}}+\mathbb{1}_{\left\{d(0, k(x+y))>B_{\alpha} \rho k\|x+y\|_{1}\right\}}+\mathbb{1}_{\left\{d(k x, k(x+y))>B_{\alpha} \rho k\|y\|_{1}\right\}}\right)\right] \\
& \leq \mathbb{P}_{p}\left(d(0, k x)>B_{\alpha} \rho k\|x\|_{1} \text { and } 0 \leftrightarrow k x\right) \\
&+\mathbb{P}_{p}\left(d(0, k(x+y))>B_{\alpha} \rho k\|x+y\|_{1} \text { and } 0 \leftrightarrow k(x+y)\right) \\
&+\mathbb{P}_{p}\left(d(k x, k(x+y))>B_{\alpha} \rho k\|y\|_{1} \text { and } k x \leftrightarrow k(x+y)\right) \\
& \leq \frac{C_{\alpha}}{k^{\alpha}}\left(\frac{1}{\|x\|_{1}^{\alpha}}+\frac{1}{\|x+y\|_{1}^{\alpha}}+\frac{1}{\|y\|_{1}^{\alpha}}\right) \text { with Lemma 2.1. }
\end{aligned}
$$

Then, using Borel-Cantelli Lemma, we know that there almost surely exists $K=K(\omega)>0$ such that for any $k \geq K$,

$$
\mathbb{1}_{A_{k}} \mathbb{1}_{\{0 \leftrightarrow \infty\}} \mathbb{1}_{\{k x \leftrightarrow \infty\}} \mathbb{1}_{\{k(x+y) \leftrightarrow \infty\}}=\mathbb{1}_{\{0 \leftrightarrow \infty\}} \mathbb{1}_{\{k x \leftrightarrow \infty\}} \mathbb{1}_{\{k(x+y) \leftrightarrow \infty\}}
$$

It follows that $\left(u_{n}\right)_{n \geq 1}$ has almost surely the same point-wise behaviour as the sequence $\left(u_{n}^{\prime}\right)_{n \geq 1}$ defined by $u_{n}^{\prime}=\frac{1}{n} \sum_{k=1}^{n} \frac{d(0, k x)}{k} \mathbb{1}_{\{0 \leftrightarrow \infty\}} \mathbb{1}_{\{k x \leftrightarrow \infty\}} \mathbb{1}_{\{k(x+y) \leftrightarrow \infty\}}$. Let us note $w_{k}=\left(\frac{d(0, k x)}{k} \mathbb{1}_{\{k x \leftrightarrow \infty\}}+\mu(x) \mathbb{1}_{\{k x \leftrightarrow \infty\})}\right) \mathbb{1}_{\{0 \leftrightarrow \infty\}}$ and $v_{k}=\mathbb{1}_{\{k x \leftrightarrow \infty\}} \mathbb{1}_{\{k(x+y) \leftrightarrow \infty\}}$. As a consequence of the convergence result in Theorem 3.2, $\left(w_{k}\right)_{k \geq 1}$ almost surely converges to $\mu(x) \mathbb{1}_{\{0 \leftrightarrow \infty\}}$. By Lemma 3.4, the Cesaro means of $\left(v_{k}\right)_{k \geq 1}$ almost surely converge to $v_{*}=\mathbb{P}_{p}(0 \leftrightarrow \infty)^{2}$.

Since $\left(v_{k}\right)_{k \geq 1}$ is a bounded sequence and $w_{k}$ converges to $w_{*}=\mu(x) \mathbb{1}_{\{0 \leftrightarrow \infty\}}$, it follows that the Cesaro means of $\left(w_{k} v_{k}\right)_{k \geq 1}$ converge to $w_{*} v_{*}=\mathbb{P}_{p}(0 \leftrightarrow \infty)^{2} \mu(x) \mathbb{1}_{\{0 \leftrightarrow \infty\}}$. (Note that

$$
\begin{aligned}
\left|\frac{1}{n} \sum_{k=1}^{n} w_{k} v_{k}-w_{*} v_{*}\right| & =\left|w_{*} \frac{1}{n} \sum_{k=1}^{n}\left(v_{k}-v_{*}\right)+\frac{1}{n} \sum_{k=1}^{n} v_{k}\left(w_{k}-w_{*}\right)\right| \\
& \leq\left|w_{*}\right| \cdot\left|\frac{1}{n} \sum_{k=1}^{n}\left(v_{k}-v_{*}\right)\right|+\|v\|_{\infty} \frac{1}{n} \sum_{k=1}^{n}\left|w_{k}-w_{*}\right|
\end{aligned}
$$

and apply Cesaro's theorem to the second term.)

This proves the desired almost-sure convergence.

To prove the convergence in mean, note that $u_{n} \leq B_{\alpha} \rho\|x\|_{1}$ and use the dominated convergence theorem.

Let us now prove the sub-additive property of $\mu$. Note first that if $x$ and $y$ are linearly dependent, the inequality is in fact an equality thanks to the homogeneity property. Now, let $x$ and $y$ be two linearly independent vectors in $\mathbb{Z}^{d}$. For every $k \geq 1$, let $A_{k}$ be the same event as the one defined in Lemma 3.5. Then, for every $k \geq 1$

$$
\begin{aligned}
\frac{d(0, k(x+y))}{k} \mathbb{1}_{A_{k}} \mathbb{1}_{\{0 \leftrightarrow \infty\}} \mathbb{1}_{\{k x \leftrightarrow \infty\}} \mathbb{1}_{\{k(x+y) \leftrightarrow \infty\}} \leq & \frac{d(0, k x)}{k} \mathbb{1}_{A_{k}} \mathbb{1}_{\{0 \leftrightarrow \infty\}} \mathbb{1}_{\{k x \leftrightarrow \infty\}} \mathbb{1}_{\{k(x+y) \leftrightarrow \infty\}} \\
& +\frac{d(k x, k(x+y))}{k} \mathbb{1}_{A_{k}} \mathbb{1}_{\{0 \leftrightarrow \infty\}} \mathbb{1}_{\{k x \leftrightarrow \infty\}} \mathbb{1}_{\{k(x+y) \leftrightarrow \infty\}}
\end{aligned}
$$


Summing for $1 \leq k \leq n$ and taking the means gives:

$$
\begin{aligned}
\mathbb{E}_{\mathbb{P}_{p}}( & \left.\frac{1}{n} \sum_{k=1}^{n} \frac{d(0, k(x+y))}{k} \mathbb{1}_{A_{k}} \mathbb{1}_{\{0 \leftrightarrow \infty\}} \mathbb{1}_{\{k x \leftrightarrow \infty\}} \mathbb{1}_{\{k(x+y) \leftrightarrow \infty\}}\right) \\
\leq & \mathbb{E}_{\mathbb{P}_{p}}\left(\frac{1}{n} \sum_{k=1}^{n} \frac{d(0, k x)}{k} \mathbb{1}_{A_{k}} \mathbb{1}_{\{0 \leftrightarrow \infty\}} \mathbb{1}_{\{k x \leftrightarrow \infty\}} \mathbb{1}_{\{k(x+y) \leftrightarrow \infty\}}\right) \\
& +\mathbb{E}_{\mathbb{P}_{p}}\left(\frac{1}{n} \sum_{k=1}^{n} \frac{d(k x, k(x+y))}{k} \mathbb{1}_{A_{k}} \mathbb{1}_{\{0 \leftrightarrow \infty\}} \mathbb{1}_{\{k x \leftrightarrow \infty\}} \mathbb{1}_{\{k(x+y) \leftrightarrow \infty\}}\right) .
\end{aligned}
$$

Thanks to Lemma $3.5,(3)$ and (4) respectively converge to $\mu(x+y) \mathbb{P}_{p}(0 \leftrightarrow \infty)^{3}$ and $\mu(x) \mathbb{P}_{p}(0 \leftrightarrow \infty)^{3}$. Moreover,

$$
\begin{aligned}
(5) & =\frac{1}{n} \sum_{k=1}^{n} \mathbb{E}_{\mathbb{P}_{p}}\left(\frac{d(k x, k(x+y))}{k} \mathbb{1}_{A_{k}} \mathbb{1}_{\{0 \leftrightarrow \infty\}} \mathbb{1}_{\{k x \leftrightarrow \infty\}} \mathbb{1}_{\{k(x+y) \leftrightarrow \infty\}}\right) \\
& =\frac{1}{n} \sum_{k=1}^{n} \mathbb{E}_{\mathbb{P}_{p}}\left(\frac{d(0, k y)}{k} \mathbb{1}_{B_{k}} \mathbb{1}_{\{0 \leftrightarrow \infty\}} \mathbb{1}_{\{-k y \leftrightarrow \infty\}} \mathbb{1}_{\{-k(x+y) \leftrightarrow \infty\}}\right),
\end{aligned}
$$

where $B_{k}$ is the event

$$
B_{k}=\left\{d(0,-k y) \leq \rho B_{\alpha} k\|y\|, d(0,-k(x+y)) \leq \rho B_{\alpha} k\|x+y\|, d(-k y,-k(x+y)) \leq \rho B_{\alpha} k\|x\|\right\}
$$

This was obtained by translating along the vector $-k x$. We use Lemma 3.5 again to conclude that (5) converges to $\mu(y) \mathbb{P}_{p}(0 \leftrightarrow \infty)^{3}$. As $\mathbb{P}_{p}(0 \leftrightarrow \infty)>0$, this leads to the desired inequality.

Step 6. Using Properties (1), (2) and (4), we get:

$$
\mu\left(u_{1}, \ldots, u_{d}\right) \leq \sum_{i=1}^{d}\left|u_{i}\right| \mu\left(e_{i}\right)
$$

where $\left(e_{i}\right)_{1 \leq i \leq d}$ is the canonical basis of $\mathbb{Z}^{d}$. Thus,

$$
\mu(u) \leq \mu_{*}\|u\|_{1}
$$

The second part of Property (3) immediately follows.

Step 7. Property (5) is a direct consequence of the sub-additivity of $\mu$.

This finally allows us to prove an analogue result for any rational direction. Indeed, let $q \in \mathbb{Q}^{d} \backslash\{0\}$ be fixed. Choose any couple $(N, u) \in \mathbb{Z}_{+}^{*} \times \mathbb{Z}^{d}$ such that $u=N q \in \mathbb{Z}^{d}$ and set $\mu(q)=\mu(u) / N$. The constant $\mu(q)$ is well defined thanks to the homogeneity property of $\mu$. The function $\mu$, extended in this manner to $\mathbb{Q}^{d} \backslash\{0\}$, obviously keeps its properties: for any rational $\alpha$ and any vectors $u$ and $v$ in $\mathbb{Q}^{d}$,

$$
\mu(\alpha u)=|\alpha| \mu(u), \text { and } \mu(u+v) \leq \mu(u)+\mu(v) \text { and }|\mu(u)-\mu(v)| \leq \rho m\|u-v\|_{1} \text {. }
$$

Thus we can extend $\mu$ to $\mathbb{R}^{d}$ by continuity, keeping these three properties. 


\section{Sufficient conditions for the positivity of $\mu$}

As in the classical case, we study now the question of the positivity of $\mu$, which is a crucial point in the quest of an asymptotic shape result. We begin with the case of a product measure.

Theorem 4.1. Assume here that $S_{\nu}=\nu^{\otimes \mathbb{E}^{d}}$. Then

- If $p \nu(0)<p_{c}$, then $\mu$ is a norm on $\mathbb{R}^{d}$.

- If $p \nu(0)>p_{c}$, then $\mu=0$ on $\mathbb{R}^{d}$.

Remark. As in the classical case, we can foresee that if $p \nu(0)=p_{c}$, then $\mu=0$ on $\mathbb{R}^{d}$. If we follow this analogy, we can guess that an easy proof would need the continuity of the time constant relatively to the distribution of the passage times. We got the feeling that such a result needs more than a few lines. So we prefer omitting the specific case $p \nu(0)=p_{c}$.

Proof. The only remaining point to consider is to determine whether $\mu(u)=0$ implies $u=0$ or not. Let us define, for each $e \in \mathbb{E}^{d}, \eta_{e}^{\prime}=\eta_{e} \omega_{e}+\left(1-\omega_{e}\right)$. The law of $\left(\eta_{e}^{\prime}\right)_{e \in \mathbb{E}^{d}}$ under $\mathbb{P}$ is $\left(p \nu+(1-p) \delta_{1}\right)^{\otimes \mathbb{E}^{d}}$. We can define the new distance relatively to these new passage times:

$$
d^{\prime}(x, y)(\omega, \eta)=\inf _{\gamma} \sum_{e \in \gamma} \eta_{e}^{\prime}
$$

where the infinimum is taken over all the paths from $x$ to $y$ in $\mathbb{Z}^{d}$. This is in fact the classical first-passage percolation distance associated to the passage times $\nu^{\prime}=p \nu+(1-p) \delta_{1}$. Denote by $\mu^{\prime}$ its associated renormalized limit. Note that by construction $d^{\prime}(x, y) \leq d(x, y)$, and thus that $\mu^{\prime} \leq \mu$. It is a classical result (see Kesten [13] for instance) that $\nu^{\prime}(0)<p_{c}$ implies that the associated limit $\mu^{\prime}$ is a norm on $\mathbb{R}^{d}$. Since $\nu^{\prime}(0)=p \nu(0)$, if $p \nu(0)<p_{c}$ then $\mu$ is a norm on $\mathbb{R}^{d}$.

On the other hand, assume that $p \nu(0)>p_{c}$. Color in red the edges $e$ such that $\omega(e)=1$ and $\nu(e)=0$. As $p \nu(0)>p_{c}$, there is almost surely a unique infinite cluster of red edges, included in the infinite cluster for the Bernoulli $(p)$ percolation structure. Let $u \in \mathbb{Z}^{d}$. On the event $R=$ "the origin is in the red infinite cluster" (included in the event "the origin is in the Bernoulli $(p)$ infinite cluster"), we can find an increasing sequence of (random) integers $\left(k_{n}\right)$ such that $k_{n} . u$ is in the red infinite cluster for every $n$. It is obvious that $d\left(0, k_{n} . u\right)=0$. Thus, evaluating $\mu(u)$ on this subsequence gives $\mu(u)=0$ on the event $R$. Since $\mu(u)$ is constant under $\overline{\mathbb{P}}$, this completes the proof.

Corollary 4.2. The function $\mu$ associated to the chemical distance in Bernoulli percolation with parameter $p>p_{c}(d)$ is a norm on $\mathbb{R}^{d}$.

When $S_{\nu}$ is not a product measure, there is no general method to determinate whether $\nu$ is a norm or not, even in the classical case $p=1$.

A natural idea is to use a stochastic comparison: if $S_{\nu^{\prime}} \succ S_{\nu}$, then $\mu\left(p, \nu^{\prime}\right) \geq \mu(p, \nu)$. Consequently, if $a$ is such that $S_{\nu^{\prime}}\left(\eta_{e} \in[a,+\infty)\right)=1$ holds for each $e \in \mathbb{E}^{d}$, then $S_{\nu^{\prime}}$ is a norm for each $p>p_{c}$, because $d(.,.) \geq a D(.,$.$) .$

We will now prove that $\mu$ is a norm if $S_{\nu}$ satisfies to an appropriate large deviation inequality.

Theorem 4.3. Let us assume that there exists $A>0$ and a map $f:(0,+\infty) \rightarrow \mathbb{R}^{+}$

$$
\forall \varepsilon>0 \quad \forall \Lambda \subseteq \mathbb{E}^{d} \quad S_{\nu}\left(\eta \in \Omega_{S} ; \sum_{e \in \Lambda} \eta_{e} \leq \varepsilon|\Lambda|\right) \leq A \exp (-f(\varepsilon)|\Lambda|) .
$$

Let $K_{0}=\varliminf_{\varepsilon \rightarrow 0^{+}} \exp (-f(\varepsilon))$.

Then, the application $\mu$ associated to $S_{\nu}$ is a norm as soon as $K_{0} p \lambda(d)<1$, where $\lambda(d)$ is the d-dimensional connective constant. 
Proof. Let $\varepsilon>0$ be small enough to ensure that $(\lambda(d)+\varepsilon) p \exp (-f(\varepsilon))<1$ and consider $u \in \mathbb{Z}^{d}$.

We have

$$
\mathbb{P}\left(d(0, u) \leq \varepsilon\|u\|_{1}\right)=\bigcup_{\Lambda \in \Lambda_{u}}\left\{\forall e \in \Lambda \quad \omega_{e}=1 \quad \text { and } \sum_{e \in \Lambda} \eta_{e} \leq \varepsilon\|u\|_{1}\right\}
$$

where $\Lambda_{u}$ is the family of subsets of $\mathbb{E}^{d}$ which are the support of a self-avoiding path from 0 to $u$. Then,

$$
\begin{aligned}
\mathbb{P}\left(d(0, u) \leq \varepsilon\|u\|_{1}\right) & \leq \sum_{\Lambda \in \Lambda_{u}} p^{|\Lambda|} \mathbb{P}\left(\sum_{e \in \Lambda} \eta_{e} \leq \varepsilon\|u\|_{1}\right) \\
& \leq \sum_{\Lambda \in \Lambda_{u}} p^{|\Lambda|} \mathbb{P}\left(\sum_{e \in \Lambda} \eta_{e} \leq \varepsilon|\Lambda|\right) \\
& \leq \sum_{n=\|u\|_{1}}^{+\infty} p^{n} A(\lambda(d)+\varepsilon)^{n} \exp (-n f(\varepsilon)) \\
& \leq \frac{A}{1-((\lambda(d)+\varepsilon) p \exp (-f(\varepsilon))}((\lambda(d)+\varepsilon) p \exp (-f(\varepsilon)))^{\|u\|_{1}} .
\end{aligned}
$$

Then, it follows from Borel-Cantelli lemma that $d(0, n u) \geq n\|u\|_{1}$ for all $n$ large enough: we can conclude that $\mu(u) \geq \varepsilon\|u\|_{1}$. As $\mu$ is homogeneous and continuous, this inequality is extended first to $\mathbb{Q}^{d}$, and next to $\mathbb{R}^{d}$. Since $\|\cdot\|_{1}$ is a norm, so does $\mu$.

Remarks. 1. We will give in Section 7.2 an explicit example where this theorem applies.

2. Let us now discuss the assumption of Theorem 4.3 by considering the case $S_{\nu}=(\operatorname{Ber}(1-q))^{\otimes \mathbb{E}^{d}}$. By Cramer's theorem, one can take

$$
f(\varepsilon)=(1-\varepsilon) \log \frac{1-\varepsilon}{q}+\varepsilon \log \frac{\varepsilon}{1-q},
$$

thus $K_{0}=q$. Then, Theorem 4.3 says that $\mu$ is a norm as soon as $q p<\frac{1}{\lambda(d)}$, whereas Theorem 4.1 ensures that the optimal condition is $q p<p_{c}$.

Since $\frac{1}{\lambda(d)} \sim \frac{1}{2 d} \sim p_{c}(d)$ for large $d$, assumption of Theorem 4.3 is not all bad.

\section{THE ASYMPtotic SHAPE THEOREM}

From now on, we assume that $\mu$ is a norm. We want now to study the convergence of the renormalized set of wet vertices at time $t$ to the unit ball for $\mu$. Because of the presence of holes in the infinite cluster, the Hausdorff distance seems naturally adapted to study this convergence.

Definition 5.1. For $x \in \mathbb{R}^{d}$ and $r \geq 0$, we define

$$
B_{\mu}(x, r)=\left\{y \in \mathbb{R}^{d} ; \mu(x-y) \leq r\right\} .
$$

Definition 5.2. The Hausdorff distance between two non empty compact subsets $A$ and $B$ of $\mathbb{R}^{d}$ is defined by

$$
\mathcal{D}(A, B)=\inf \left\{r \geq 0 ; A \subset B+B_{\mu}(0, r) \text { and } B \subset A+B_{\mu}(0, r)\right\} .
$$

Note that we use the Hausdorff distance associated to $\mu$, but the equivalence of norms on $\mathbb{R}^{d}$ ensures that the induced topology does not depend on this choice. 
Theorem 5.3 (asymptotic shape). Assume that $\left(H_{\alpha}\right)$ holds for some $\alpha>d^{2}+2 d-1$ and that $\mu$ is a norm. Then,

$$
\lim _{t \rightarrow+\infty} \mathcal{D}\left(\frac{B_{t}}{t}, B_{\mu}(0,1)\right)=0 \quad \overline{\mathbb{P}} \text { a.s. }
$$

Remark. Recall that $\left(H_{\alpha}\right)$ is always satisfied if the passage times are bounded by an absolute constant. We have already seen that $S_{\nu}$ is always a norm when the passage times are bounded from below by a positive constant. Putting these two results together, we get that $S_{\nu}$ always satisfies the assumptions of Theorem 5.3 if $S_{\nu}$ is an invariant ergodic measure which satisfies to $S_{\nu}\left([a, b]^{\mathbb{E}^{d}}\right)=1$ for some $a, b$ with $0<a<b<+\infty$. We thus obtain an asymptotic shape theorem for the chemical distance in supercritical Bernoulli percolation.

Corollary 5.4 (asymptotic shape for chemical distance in supercritical Bernoulli percolation). Remember that on the event "the origin is in the infinite cluster", we denote by $B_{n}$ the random set of vertices in $\mathbb{Z}^{d}$ whose chemical distance from 0 is less or equal to $n$.

For every $d \geq 2$ and $p>p_{c}(d)$, there exists a deterministic convex compact set $A \subset \mathbb{R}^{d}$ with non-empty interior and invariant under permutations or reflections of the coordinates in $\mathbb{R}^{d}$ such that:

$$
\frac{B_{n}}{n} \rightarrow A \text { for the Hausdorff topology } \overline{\mathbb{P}}_{p} \text { a.s. }
$$

We will first give some preliminary lemmas in order to prove Theorem 5.3. The first one gives the existence of vertices of the infinite cluster in any long enough "integer segment line" in the plane:

Lemma 5.5. Let $z \in \mathbb{Z}^{d}$ be a fixed non null vector. Then for every $\varepsilon>0$,

$$
\overline{\mathbb{P}}\left(\exists N>0, \forall r \in[N,+\infty), \exists k \in[(1-\varepsilon) r, \ldots,(1+\varepsilon) r] \cap \mathbb{Z}_{+} \text {such that } k z \leftrightarrow \infty\right)=1
$$

Proof. Almost surely under $\overline{\mathbb{P}}$, the sequence $\left(T_{m, z}\right)_{m \geq 1}$ is an unbounded increasing sequence; thus there exists $m \in \mathbb{Z}_{+}$, with $T_{m, z} \leq r<T_{m+1, z}$. Now,

$$
0 \leq \frac{r-T_{m, z}}{r} \leq \frac{T_{m+1, z}-T_{m, z}}{T_{m, z}}=\frac{T_{m+1, z}}{T_{m, z}}-1=\frac{m+1}{m} \frac{\frac{T_{m+1, z}}{m+1}}{\frac{T_{m, z}}{m}}-1,
$$

which vanishes at infinity since $\frac{T_{m, z}}{m}$ tends to $\frac{1}{\mathbb{P}(0 \leftrightarrow \infty)}$. It follows that $\frac{r-T_{m, z}}{r} \leq \varepsilon$ as soon as $m$ is large enough, thus we can take $k=T_{m, z}$.

The second lemma ensures that a finite travel time $d(x, y)$ between two vertices that are close enough from each other cannot be too large, provided that $x$ and $y$ are sufficiently far from the origin.

Lemma 5.6. Assume that $\left(H_{\alpha}\right)$ holds with $\alpha>d^{2}+2 d-1$. Then for all $\varepsilon>0$,

$$
\mathbb{P}\left(\exists M>0, \forall m \geq M, \forall x \in \mathbb{Z}^{d}, \forall y \in \mathbb{Z}^{d},\left(\|x\|_{1}=m,\|y-x\|_{1} \leq \varepsilon m, x \leftrightarrow y\right) \Rightarrow d(x, y) \leq B_{\alpha} \rho \varepsilon m\right)=1
$$

Proof. In view to use Borel-Cantelli lemma, denote by $A_{m}$ the set:

$$
A_{m}=\left\{\exists x \in \mathbb{Z}^{d}, \exists y \in \mathbb{Z}^{d} \text { such that }\|x\|_{1}=m,\|y-x\|_{1} \leq \varepsilon m, x \leftrightarrow y \text { and } d(x, y) \geq B_{\alpha} \rho \varepsilon m\right\}
$$

Considering Lemmas 2.3 and 2.1, we note $a_{\alpha}, C_{\alpha}, D_{\alpha}$ the constants that are given by these lemmas. Note that in the following calculations, $K_{i}$ and $L_{j}$ denote constants, depending only on the dimension $d$ and on $\alpha$, whose values are not specified to avoid intricate expressions. 
We set $r=\frac{\left(B_{\alpha} \rho \varepsilon m\right)^{1 / d}}{a_{\alpha}}$.

$$
\begin{aligned}
\mathbb{P}\left(A_{m}\right) \leq & \sum_{x,\|x\|_{1}=m} \sum_{l=0}^{\varepsilon m} \sum_{y,\|x-y\|_{1}=l} \mathbb{P}\left(x \leftrightarrow y, d(x, y) \geq B_{\alpha} \rho \varepsilon m\right) \\
\leq & \sum_{x,\|x\|_{1}=m} \sum_{l=0}^{r} \sum_{y,\|y\|_{1}=l} \mathbb{P}\left(0 \leftrightarrow y, d(0, y) \geq B_{\alpha} \rho \varepsilon m\right) \\
& +\sum_{x,\|x\|_{1}=m} \sum_{l=r}^{\varepsilon m} \sum_{y,\|y\|_{1}=l} \mathbb{P}\left(y \leftrightarrow 0, d(0, y) \geq B_{\alpha} \rho\|y\|_{1}\right) \\
\leq & \sum_{x,\|x\|_{1}=m} \sum_{l=0}^{r} \sum_{y,\|y\|_{1}=l} \frac{D_{\alpha}}{r^{\nu}} \quad \text { (by Lem. 2.3) } \\
& +\sum_{x,\|x\|_{1}=m} \sum_{l=r}^{\varepsilon m} \sum_{y,\|y\|_{1}=l} \frac{C_{\alpha}}{l^{\alpha}} \quad \text { (by Lem. 2.1). }
\end{aligned}
$$

Let us compute the first term

$$
\begin{aligned}
\sum_{x,\|x\|_{1}=m} \sum_{l=0}^{r} \sum_{y,\|y\|_{1}=l} \frac{D_{\alpha}}{r^{\nu}} & \leq K_{1} \sum_{x,\|x\|_{1}=m} \sum_{l=0}^{r} l^{d-1} \frac{1}{r^{\nu}} \\
& \leq K_{2} \sum_{x,\|x\|_{1}=m} \frac{r^{d}}{r^{\nu}} \\
& \leq K_{3} \frac{1}{m^{\frac{\nu}{d}-d}},
\end{aligned}
$$

and the second term:

$$
\begin{aligned}
\sum_{x,\|x\|_{1}=m} \sum_{l=r}^{\varepsilon m} \sum_{y,\|y\|_{1}=l} \frac{C_{\alpha}}{l^{\alpha}} & \leq L_{1} \sum_{x,\|x\|_{1}=m} \sum_{l=r}^{\varepsilon m} l^{d-1} \frac{1}{l^{\alpha}} \\
& \leq L_{1} \sum_{x,\|x\|_{1}=m} \sum_{l=r}^{+\infty} l^{d-1} \frac{1}{l^{\alpha}} \\
& \leq L_{2} \sum_{x,\|x\|_{1}=m} \frac{1}{m^{(\alpha-d) / d}} \\
& \leq L_{3} \frac{1}{m^{\frac{\alpha-d}{d}-d+1}} .
\end{aligned}
$$

Remember that $\nu=\alpha-d+1$. The choice that we made on $\alpha$ ensures that both parts are the general terms of convergent series, which allows us to conclude thanks to Borel-Cantelli lemma.

The last lemma proves the uniform convergence of the travel times to the directional asymptotic time constants in the considered direction:

Lemma 5.7. Assume that $\left(H_{\alpha}\right)$ holds with $\alpha>d^{2}+2 d-1$. Then for all $\varepsilon>0$,

$$
\overline{\mathbb{P}}\left(\exists M>0, \forall y \in \mathbb{Z}^{d},\left(\|y\|_{1} \geq M \text { and } y \leftrightarrow 0\right) \Rightarrow|d(0, y)-\mu(y)| \leq \varepsilon\|y\|_{1}\right)=1 .
$$


Proof. Let us assume this is false and choose $\varepsilon>0$ such that the assertion fails. Then, with positive probability under $\overline{\mathbb{P}}$, there exists a random sequence $\left(y_{n}\right)_{n \geq 0}$ of vertices in $\mathbb{Z}^{d}$ such that

$$
\left\{\begin{array}{l}
\left\|y_{n}\right\|_{1} \rightarrow \infty \\
y_{n} \leftrightarrow 0 \\
\left|d\left(0, y_{n}\right)-\mu\left(y_{n}\right)\right| \geq \varepsilon\left\|y_{n}\right\|_{1} .
\end{array}\right.
$$

Considering a subsequence if necessary, assume that

$$
\frac{y_{n}}{\left\|y_{n}\right\|_{1}} \rightarrow z
$$

Let us approximate $z$ by a renormalized integer vector: consider $\varepsilon_{1}>0$ (to be chosen small enough later), and choose $z^{\prime} \in \mathbb{Z}^{d}$ be such that $\left\|\frac{z^{\prime}}{\left\|z^{\prime}\right\|_{1}}-z\right\|_{1} \leq \varepsilon_{1}$.

Let us find, for each $y_{n}$, an integer point on the line $\mathbb{R} z^{\prime}$ close enough from $y_{n}$ : let $h_{n}$ be the integer part of $\frac{\left\|y_{n}\right\|_{1}}{\left\|z^{\prime}\right\|_{1}}$. We have

$$
\begin{aligned}
\left\|y_{n}-h_{n} . z^{\prime}\right\|_{1} & \leq\left\|y_{n}-\frac{\left\|y_{n}\right\|_{1}}{\left\|z^{\prime}\right\|_{1}} z^{\prime}\right\|_{1}+\left|\frac{\left\|y_{n}\right\|_{1}}{\left\|z^{\prime}\right\|_{1}}-h_{n}\right|\left\|z^{\prime}\right\|_{1} \\
& \leq\left\|y_{n}\right\|_{1}\left\|\frac{y_{n}}{\left\|y_{n}\right\|_{1}}-\frac{z^{\prime}}{\left\|z^{\prime}\right\|_{1}}\right\|_{1}+\left\|z^{\prime}\right\|_{1} .
\end{aligned}
$$

Choose $N>0$ large enough to ensure that $(n \geq N) \Rightarrow\left(\left\|\frac{y_{n}}{\left\|y_{n}\right\|_{1}}-z\right\|_{1} \leq \varepsilon_{1}\right)$.

With the choice we made for $z^{\prime}$, we have $(n \geq N) \Rightarrow\left(\left\|\frac{y_{n}}{\left\|y_{n}\right\|_{1}}-\frac{z^{\prime}}{\left\|z^{\prime}\right\|_{1}}\right\|_{1} \leq 2 \varepsilon_{1}\right)$, whence

$$
\left\|y_{n}-h_{n} \cdot z^{\prime}\right\|_{1} \leq 2 \varepsilon_{1}\left\|y_{n}\right\|_{1}+\left\|z^{\prime}\right\|_{1}
$$

Enlarging $N$ if necessary, Lemma 5.5 ensures now the existence, for each $n \geq N$, of an integer $k_{n} \in[(1-$ $\left.\left.\varepsilon_{1}\right) h_{n}, \ldots,\left(1+\varepsilon_{1}\right) h_{n}\right]$ such that $k_{n} z^{\prime}$ is in the infinite cluster. But:

$$
\begin{aligned}
\left\|y_{n}-k_{n} . z^{\prime}\right\|_{1} & \leq\left\|y_{n}-h_{n} . z^{\prime}\right\|_{1}+\left|h_{n}-k_{n}\right|\left\|z^{\prime}\right\|_{1} \\
& \leq 2 \varepsilon_{1}\left\|y_{n}\right\|_{1}+\left\|z^{\prime}\right\|_{1}+\varepsilon_{1} h_{n}\left\|z^{\prime}\right\|_{1} \\
& \leq 2 \varepsilon_{1}\left\|y_{n}\right\|_{1}+\left\|z^{\prime}\right\|_{1}+\varepsilon_{1}\left\|y_{n}\right\|_{1} \\
& \leq 3 \varepsilon_{1}\left\|y_{n}\right\|_{1}+\left\|z^{\prime}\right\|_{1} \\
& \leq 4 \varepsilon_{1}\left\|y_{n}\right\|_{1}
\end{aligned}
$$

if $N$ is large enough. Enlarging once again $N$ if necessary, we can use Lemma 5.6 to have:

$$
\forall n \geq N \quad d\left(y_{n}, k_{n} \cdot z^{\prime}\right) \leq 4 B_{\alpha} \rho \varepsilon_{1}\left\|y_{n}\right\|_{1} .
$$

Finally, for every $n$ large enough, we have:

$$
\begin{aligned}
\left|d\left(0, y_{n}\right)-\mu\left(y_{n}\right)\right| & \leq\left|d\left(0, y_{n}\right)-d\left(0, k_{n} \cdot z^{\prime}\right)\right|+\left|d\left(0, k_{n} \cdot z^{\prime}\right)-\mu\left(k_{n} \cdot z^{\prime}\right)\right|+\left|\mu\left(k_{n} \cdot z^{\prime}\right)-\mu\left(y_{n}\right)\right| \\
& \leq 4 B_{\alpha} \rho \varepsilon_{1}\left\|y_{n}\right\|_{1}+k_{n}\left|\frac{d\left(0, k_{n} \cdot z^{\prime}\right)}{k_{n}}-\mu\left(z^{\prime}\right)\right|+\mu_{1}\left\|k_{n} \cdot z^{\prime}-y_{n}\right\|_{1} \\
& \leq 4 B_{\alpha} \rho \varepsilon_{1}\left\|y_{n}\right\|_{1}+\left(1+\varepsilon_{1}\right) \frac{\left\|y_{n}\right\|_{1}}{\left\|z^{\prime}\right\|_{1}}\left|\frac{d\left(0, k_{n} \cdot z^{\prime}\right)}{k_{n}}-\mu\left(z^{\prime}\right)\right|+4 \varepsilon_{1} \mu_{1}\left\|y_{n}\right\|_{1} .
\end{aligned}
$$


But the convergence in the direction given by $z^{\prime}$ ensures that for every $n$ large enough,

$$
\left|\frac{d\left(0, k_{n} \cdot z^{\prime}\right)}{k_{n}}-\mu\left(z^{\prime}\right)\right| \leq \varepsilon_{1}
$$

If we choose $\varepsilon_{1}$ small enough, we can ensure that for every $n$ large enough,

$$
\left|d\left(0, y_{n}\right)-\mu\left(y_{n}\right)\right|<\varepsilon\left\|y_{n}\right\|_{1},
$$

which yields to a contradiction.

The proof of Theorem 5.3 is now just a consequence of these lemmas:

Proof of Theorem 5.3. Let $\varepsilon>0$.

Step 1. If

$$
\exists T>0 \quad \forall t \geq T \quad \frac{B_{t}}{t} \subset B_{\mu}(0,(1+\varepsilon))
$$

fails, then there exists an unbounded increasing sequence of times $\left(t_{n}\right)_{n \geq 1}$ and a sequence of vertices $\left(y_{n}\right)_{n \geq 1}$ with $y_{n} \in B\left(t_{n}\right)$ and $\frac{y_{n}}{t_{n}} \notin B_{\mu}(0,1+\varepsilon)$, which gives $d\left(0, y_{n}\right) \leq t_{n}$ and $\mu\left(y_{n}\right)>(1+\varepsilon) t_{n}$; this leads to:

$$
\mu\left(y_{n}\right)-d\left(0, y_{n}\right) \geq\left(1-\frac{1}{1+\varepsilon}\right) \mu\left(y_{n}\right)
$$

But, since $\mu\left(y_{n}\right) \geq(1+\varepsilon) t_{n}$ and $\mu$ is a norm, the sequence $\left(\left\|y_{n}\right\|\right)_{n \in \mathbb{Z}_{+}}$goes to infinity. By Lemma 5.7 , this can only happen with a null probability.

Step 2. Let us show that with probability 1, the following property holds:

$$
\exists T>0 \quad \forall t \geq T \quad B_{\mu}\left(0,1-\frac{\varepsilon}{2}\right) \subset \frac{B_{t}}{t}+B_{\mu}\left(0, \frac{\varepsilon}{2}\right)
$$

If (6) fails, then, with positive probability under $\overline{\mathbb{P}}$, there exists an unbounded increasing sequence of random times $\left(t_{n}\right)_{n \geq 1}$ and a sequence $\left(v_{n}\right)_{n \geq 1}$ of points in $\mathbb{R}^{d}$ such that

$$
\left\{\begin{array}{l}
v_{n} \in B_{\mu}\left(0,1-\frac{\varepsilon}{2}\right) \\
v_{n} \notin \frac{B_{t_{n}}}{t_{n}}+B_{\mu}\left(0, \frac{\varepsilon}{2}\right) .
\end{array}\right.
$$

Let $v$ be a limiting value for $\left(v_{n}\right)_{n \geq 1}$ : clearly, there exists an unbounded increasing sequence of times $t_{n}^{\prime}$ such that $v \notin \frac{B_{t_{n}^{\prime}}}{t_{n}^{\prime}}+B_{\mu}\left(0, \frac{\varepsilon}{3}\right)$. Let $w \in \mathbb{Q}^{d}$ such that $\mu(v-w) \leq \frac{\varepsilon}{12}$. Then, for each $n \geq 1$, we have $w \notin \frac{B_{t_{n}^{\prime}}}{t_{n}^{\prime}}+B_{\mu}\left(0, \frac{\varepsilon}{4}\right)$, whence $w \in B_{\mu}\left(0,1-\frac{5}{12} \varepsilon\right)$. We can write $w$ as $w=z / Z$, with $z \in \mathbb{Z}^{d}$ and $Z \in \mathbb{Z}_{+}$.

Provided that $n$ is large enough, Lemma 5.5 ensures that, there exists $z_{n} \in B_{\mu}\left(t_{n}^{\prime} w, \frac{\varepsilon}{4} \mu\left(t_{n}^{\prime} w\right)\right)$ with $z_{n} \leftrightarrow \infty$. The vertex $z_{n}$ can not be in $B_{t_{n}^{\prime}}$ because $w \notin \frac{B_{t_{n}^{\prime}}}{t_{n}^{\prime}}+B_{\mu}\left(0, \frac{\varepsilon}{4}\right)$. It follows that $d\left(0, z_{n}\right)>t_{n}^{\prime}$. But

$$
\mu\left(z_{n}\right) \leq \mu\left(t_{n}^{\prime} w\right)+\frac{\varepsilon}{4} \mu\left(t_{n}^{\prime} w\right) \leq t_{n}^{\prime} \mu(w)+\frac{\varepsilon}{4} t_{n}^{\prime} \leq t_{n}^{\prime}\left(1-\frac{5 \varepsilon}{12}+\frac{\varepsilon}{4}\right)=\left(1-\frac{\varepsilon}{6}\right) t_{n}^{\prime} .
$$

By Lemma 5.7, this can (almost surely) not happen. Then, (6) holds almost surely. Since

$$
B_{\mu}\left(0,1-\frac{\varepsilon}{2}\right) \subset \frac{B_{t}}{t}+B_{\mu}\left(0, \frac{\varepsilon}{2}\right) \Longrightarrow B_{\mu}(0,1) \subset \frac{B_{t}}{t}+B_{\mu}(0, \varepsilon),
$$

this completes the proof. 


\section{Properties of the Asymptotic Shape}

As in the classical case, one has immediate properties of stochastic comparison for the asymptotic shape (see Kesten [13], Chap. 6):

Lemma 6.1 (stochastic comparison). Let $p_{c}<p \leq p^{\prime}$ and $\nu, \nu^{\prime}$ be two probability measures on $\mathbb{R}^{+}$such that the assumptions of the shape theorem are fulfilled for $S_{\nu}=\nu^{\otimes \mathbb{E}^{d}}$ and $S_{\nu^{\prime}}=\left(\nu^{\prime}\right)^{\otimes \mathbb{E}^{d}}$ and such that $\nu^{\prime}$ is stochastically smaller than $\nu$, then if $A$ and $A^{\prime}$ denote their respective associated asymptotic shapes, we have

$$
A \subset A^{\prime} .
$$

Proof. It is just a classical coupling argument.

Lemma 6.2. Let $p>p_{c}$, and $\nu$ be a probability measure on $\mathbb{R}^{+}$satisfying the assumptions of the shape theorem. Denote by $\nu_{\min }$ the essential infimum of $\nu, \nu_{\text {mean }}$ the mean of $\nu$, by $\mu_{p, \nu}$ the norm associated to the first passage-percolation model with $S_{\nu}=\nu^{\otimes \mathbb{E}^{d}}$ in the random environment given by the infinite cluster of Bernoulli $(p)$ percolation, and by $\tilde{\mu}_{p}$ the norm associated to the chemical distance for Bernoulli $(p)$ percolation. Then:

$$
\forall y \in \mathbb{Z}^{d} \quad \nu_{\min } \tilde{\mu}_{p}(y) \leq \mu_{p, \nu}(y) \leq \nu_{\text {mean }} \tilde{\mu}_{p}(y) .
$$

Proof. The lower bound is obtained by a coupling argument, the upper one by estimating the travel times on the minimal paths for the chemical distance.

The next theorem gives the existence of a flat edge for the asymptotic shape under some conditions on $p$ and the distribution of the passage times, and determine the exact length of this flat edge - see Durrett-Liggett [9] Lemmas 6-13 and Marchand [15] Theorem 1.3 for the analogous result in the classical setting.

Theorem 6.3 (flat edge result). Here, we work on $\mathbb{Z}^{2}$. Let $p>p_{c}=1 / 2$. Denote by $\overrightarrow{p_{c}}$ the critical threshold for oriented percolation on $\mathbb{Z}^{2}$. Assume that $S_{\nu}=\nu^{\otimes \mathbb{E}^{2}}$, where $\nu$ is a probability measure on $\mathbb{R}^{+}$such that $\nu_{\mathrm{min}}$, the essential infimum of $\nu$, is positive, satisfies the assumption of the shape theorem. Denote by $A_{p}$ the associated asymptotic shape, and by $\diamond(r)$ the ball with radius $r$ for $\|.\|_{1}$.

- $A_{p} \subset \diamond\left(\frac{1}{\nu_{\min }}\right)$.

- If $p \nu\left(\nu_{\min }\right)<\overrightarrow{p_{c}}$, then $A_{p} \subset$ int $\left(\diamond\left(\frac{1}{\nu_{\min }}\right)\right)$.

- If $p \nu\left(\nu_{\min }\right)>\overrightarrow{p_{c}}$, then

$$
A_{p} \cap \operatorname{fr}\left(\diamond\left(\frac{1}{\nu_{\min }}\right)\right) \cap\left(\mathbb{R}^{+}\right)^{2}=\left[\frac{1}{\nu_{\min }} M_{q}, \frac{1}{\nu_{\min }} N_{q}\right],
$$

where

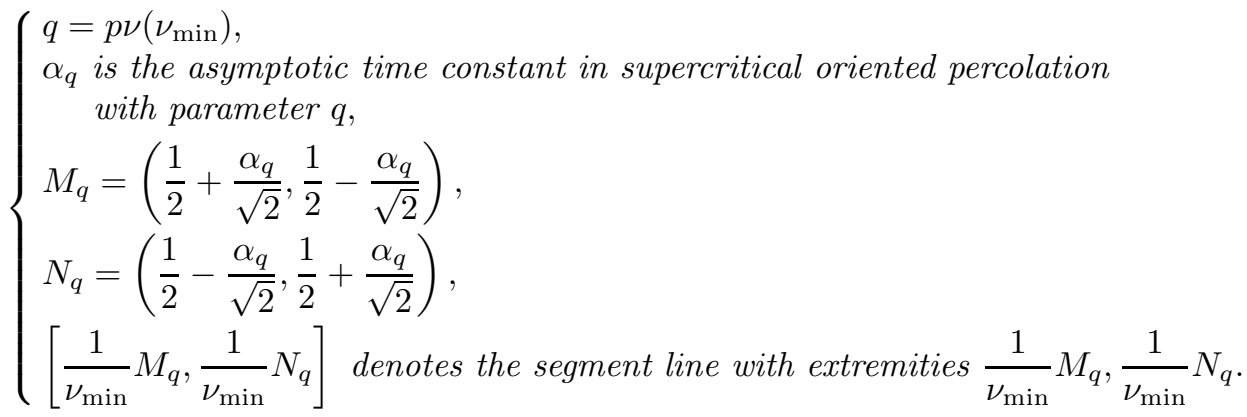


Corollary 6.4 (flat edge for the asymptotic shape for the chemical distance). Here, we work on $\mathbb{Z}^{2}$. Let $p>p_{c}=1 / 2$. Denote by $\overrightarrow{p_{c}}$ the critical threshold for oriented percolation on $\mathbb{Z}^{2}$. Denote by $A_{p}$ the associated asymptotic shape, and by $\diamond$ the unit ball with radius 1 for $\|.\|_{1}$.

- $A_{p} \subset \diamond$.

- If $p<\overrightarrow{p_{c}}$, then $A_{p} \subset$ int $(\diamond)$.

- If $p>\overrightarrow{p_{c}}$, then

$$
A_{p} \cap \operatorname{fr}(\diamond) \cap\left(\mathbb{R}^{+}\right)^{2}=\left[M_{p}, N_{p}\right],
$$

with the same notations as in Theorem 6.3.

The following pictures represent realizations of the set $B_{n}$ for the chemical distance. The simulation is stopped as soon as the process goes out from a $800 \times 800$ box. In each picture, the color of a point is determined by its wetting time. Note the absence of flat edge for $p=0.55<\overrightarrow{p_{c}}$, and the flat edges for $p=0.7>\overrightarrow{p_{c}}$.
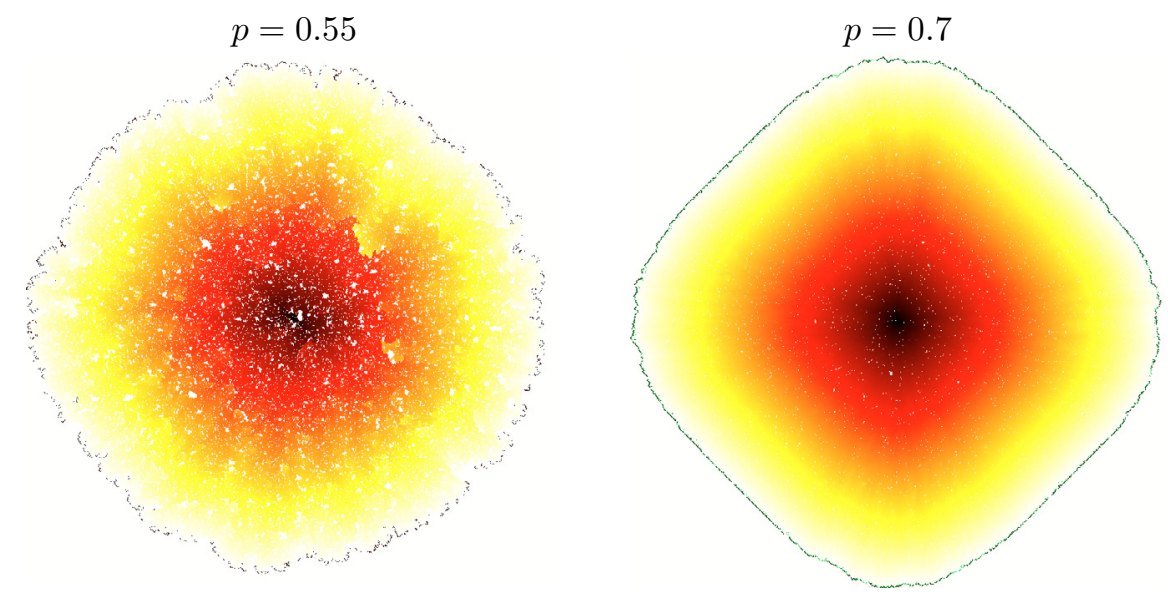

Proof of Theorem 6.3. The proof is really similar to the one of the analogous result in the general case (see Durrett-Liggett [9] Lems. 6-13 and Marchand [15] Th. 1.3). Thus we only give a quick proof, mainly based on the arguments given in these papers and on stochastic comparison.

Note that the first point is a direct consequence of Lemma 6.1, by comparing our model with the classical case and passage times with law $\delta_{\nu_{\min }}$.

Note then, by Lemma 6.1, that the asymptotic shape of the first-passage percolation for some $\nu^{\otimes \mathbb{E}^{2}}$ on the infinite cluster of $\operatorname{Bernoulli}(p)$ percolation is included in the asymptotic shape of classical first-passage percolation for the same $\nu^{\otimes \mathbb{E}^{2}}$. Thus the second point is a consequence of Theorem 1.3 (ii) in Marchand [15], which is itself a direct consequence of the strict inequalities for the time constant in classical first-passage percolation established by van den Berg and Kesten [19].

For the third point, let us consider first the proof of the inclusion

$$
A_{p} \cap \operatorname{fr}\left(\diamond\left(\frac{1}{\nu_{\min }}\right)\right) \cap\left(\mathbb{R}^{+}\right)^{2} \supset\left[\frac{1}{\nu_{\min }} M_{q}, \frac{1}{\nu_{\min }} N_{q}\right] .
$$

As noted in Kesten [13] in the proof of Theorem 6.13, the proof of the analogous result in the special case of Richardson's model treated in Durrett and Liggett [9] Lemmas 6-13 can immediately be adapted.

It remains now to prove the exact length of the flat edge by proving:

$$
A_{p} \cap \operatorname{fr}\left(\diamond\left(\frac{1}{\nu_{\min }}\right)\right) \cap\left(\mathbb{R}^{+}\right)^{2} \subset\left[\frac{1}{\nu_{\min }} M_{q}, \frac{1}{\nu_{\min }} N_{q}\right] .
$$


But once again, the stochastic comparison lemma and the exact determination result in the classical case Theorem 1.3 in Marchand [15] - give the announced result.

\section{EXAMPLES}

\subsection{Exponential passage times}

This subsection is devoted to the special case where the passage times are independent variables with an exponential distribution $\mathcal{E}(\lambda)$. When $p=1$, we recover a Richardson model, which is one of the first studied examples for first-passage percolation - see Richardson [16].

We want to take advantage of the scaling property of the exponential passage times to compare the asymptotic shape in a random environment $(p<1)$ and the asymptotic shape in the classical case $(p=1)$.

By Theorem 4.1, $\mu$ is a norm for $S_{\nu}=(\mathcal{E}(\lambda))^{\otimes \mathbb{E}^{d}}$ and each $p>p_{c}$. Let us denote by $A(p, \lambda)$ the asymptotic shape corresponding to $S_{\nu}=(\mathcal{E}(\lambda))^{\otimes \mathbb{E}^{d}}$. By the scaling properties of the exponential laws, it is easy to see that $A(p, \lambda)=\lambda A(p, 1)$ holds for each $\lambda>0$ and $p>p_{c}$. We would like to compare in this case the asymptotic shape for the random environment and the asymptotic shape in the classical case. Thanks to the scaling property, we only have to consider the case with exponential distribution with parameter 1.

Lemma 7.1. $A(p, 1) \subset A(1, p)=p A(1,1)$.

Proof. The main tool is an appropriate coupling. Let $\left(\eta_{e}\right)_{e \in \mathbb{E}^{d}},\left(x_{e}\right)_{e \in \mathbb{E}^{d}},\left(z_{e}\right)_{e \in \mathbb{E}^{d}}$ be independent random variables with for each $e \in \mathbb{E}^{d}: \eta_{e} \sim \mathcal{E}(p), x_{e} \sim \mathcal{E}(1-p)$ and $z_{e} \sim \mathcal{E}(1)$. Now define

$$
\eta_{e}^{\prime}=\mathbb{1}_{\left\{\eta_{e} \leq x_{e}\right\}} \eta_{e}+\mathbb{1}_{\left\{\eta_{e}>x_{e}\right\}} z_{e}
$$

and

On the one hand,

$$
\omega_{e}=\mathbb{1}_{\left\{\eta_{e} \leq x_{e}\right\}} .
$$

$$
\begin{aligned}
\mathbb{P}\left(\eta_{e}^{\prime}>t, \omega_{e}=1\right) & =\mathbb{P}\left(\eta_{e}>t, \eta_{e} \leq x_{e}\right) \\
& =\int_{t}^{+\infty} p \mathrm{e}^{-p y} \mathbb{P}\left(x_{e}>y\right) \mathrm{d} y \\
& =\int_{t}^{+\infty} p \mathrm{e}^{-p y} \mathrm{e}^{-(1-p) y} \mathrm{~d} y \\
& =p \mathrm{e}^{-t}
\end{aligned}
$$

Particularly, $\mathbb{P}\left(\omega_{e}=1\right)=\mathbb{P}\left(\eta_{e}^{\prime}>0, \omega_{e}=1\right)=p$. On the other hand

$$
\mathbb{P}\left(\eta_{e}^{\prime}>t, \omega_{e}=0\right)=\mathbb{P}\left(z_{e}>t, \eta_{e}>x_{e}\right)=\mathbb{P}\left(z_{e}>t\right) \mathbb{P}\left(\eta_{e}>x_{e}\right)=\mathrm{e}^{-t}(1-p)
$$

Thus, $\eta_{e}^{\prime}$ and $\omega_{e}$ are independent random variables, with $\eta_{e}^{\prime} \sim \mathcal{E}(1)$ and $\omega_{e} \sim \operatorname{Ber}(p)$.

Consider now the set of wet vertices $B_{t}$ and $B_{t}^{\prime}$, respectively associated to $\left(\left(1, \eta_{e}\right)_{e \in \mathbb{E}^{d}}\right)$ and $\left(\left(\omega_{e}, \eta_{e}^{\prime}\right)\right)_{e \in \mathbb{E}^{d}}$. Since $\eta_{e}^{\prime}=\eta_{e}$ when the bond $e$ is open, it follows that

$$
B^{\prime}(t) \subset B(t)
$$

always holds.

Dividing by $t$, and letting $t$ tend to infinity, we get $A(p, 1) \subset A(1, p)=p A(1,1)$. 


\subsection{Dependent $\chi^{2}$ passage times}

We then give an example of unbounded dependent passage times which lead to an asymptotic shape. It is also an example in which our Theorem 4.3 enables to prove that $\mu$ is effectively a norm.

Theorem 7.2. Let $\left(X_{n}^{i}\right)_{n \in \mathbb{Z}^{d}, i \in\{1, \ldots, d\}}$ be a centered Gaussian process such that

$$
\forall i, j \in\{1, \ldots, d\} \quad \forall k, n \in \mathbb{Z}^{d} \quad \mathbb{E} X_{n}^{i} X_{n+k}^{j}=\mathbb{E} X_{0}^{i} X_{k}^{j}
$$

and

$$
\sum_{k \in \mathbb{Z}^{d}} \sum_{1 \leq i \leq j \leq d}\left|\mathbb{E} X_{0}^{i} X_{k}^{j}\right|<+\infty .
$$

To avoid degenerate cases, we also assume that

$$
\forall i \in\{1, \ldots, d\} \quad \mathbb{E}\left(X_{0}^{i}\right)^{2}>0
$$

Now consider the passage time $\left(\eta_{e}\right)_{e \in \mathbb{E}^{d}}$ defined by $\eta_{\left\{k, k+e_{i}\right\}}=\left(X_{k}^{i}\right)^{2}$. If we denote by $S_{\nu}$ the law of $\left(\eta_{e}\right)_{e \in \mathbb{E}^{d}}$, then $S_{\nu}$ satisfies to the assumption of Theorem 5.3 for each $p>p_{c}$, so there is convergence to the asymptotic shape for the associated growth model.

Proof. It is immediate that $S_{\nu}$ is shift-invariant. Since the covariance is summable, it tends to zero at infinity; it follows that $S_{\nu}$ is mixing, hence it is ergodic - the previous result is attributed to Maruyama and Fomin by Sinaï [17].

Let us first recall some useful estimates whose proofs can be found in Garet [10].

Lemma 7.3. Let $X$ be a $\mathbb{R}^{n}$-valued centered Gaussian vector with covariance matrix $C$. Let us denote by $\rho(C)$ the spectral radius i.e. the largest norm of an eigenvalue of $C$. Then, for each $a^{2}>\rho(C)$, we have:

$$
P\left(\|X\|_{2}^{2} \geq n a^{2}\right) \leq \mathrm{e}^{-n h\left(\frac{a^{2}}{\rho(C)}\right)}
$$

where $h(x)=\frac{1}{2}(x-\ln x-1)$. The function $h$ is increasing and positive on $(1,+\infty)$, with $+\infty$ as limit at $+\infty$.

Lemma 7.4. Let $X$ be a n-dimensional Gaussian vector with positive definite covariance matrix $C$. Let us denote by $\Upsilon(C)$ the spectral gap i.e. the smallest eigenvalue of $C$. Then, for each $a^{2}<\Upsilon(C)$, we have

$$
P\left(\|X\|_{2}^{2} \leq n a^{2}\right) \leq \mathrm{e}^{-n h\left(\frac{a^{2}}{\Upsilon(C)}\right)},
$$

where $h(x)=\frac{1}{2}(x-\ln x-1)$. The function $h$ is positive and decreasing on $(0,1)$, with an infinite limit at 0 .

We will now prove that $\left(\eta_{e}\right)_{e \in \mathbb{E}^{d}}$ satisfy to the assumption of Theorem 4.3.

Let us for convenience identify $\mathbb{E}^{d}$ with $\mathbb{Z}^{d} \times\{1, \ldots, d\}$ : the point $(x, i) \in \mathbb{Z}^{d} \times\{1, \ldots d\}$ will be identified with $\left\{x, x+e_{i}\right\} \in \mathbb{E}^{d}$. We define

$$
S=\sum_{k \in \mathbb{Z}^{d}} \sum_{1 \leq i \leq j \leq d}\left|\mathbb{E} X_{0}^{i} X_{k}^{j}\right|<+\infty \text { and } \sigma^{2}=\inf \left\{\mathbb{E}\left(X_{0}^{i}\right)^{2}>0 ; i \in\{1, \ldots, d\}\right\}
$$

Let $\Lambda \subseteq \mathbb{Z}^{d} \times\{1, \ldots d\}$ and $\varepsilon>0$. We must bound

$$
S_{\nu}\left(\eta \in \Omega_{S} ; \sum_{e \in \Lambda} \eta_{e} \leq \varepsilon|\Lambda|\right)=\mathbb{P}\left(\sum_{(k, i) \in \Lambda}\left(X_{k}^{i}\right)^{2} \leq \varepsilon|\Lambda|\right)
$$


Since $\sum_{k \in N \mathbb{Z}^{d} \backslash\{0\}} \sum_{1 \leq i \leq j \leq d}\left|\mathbb{E} X_{0}^{i} X_{k}^{j}\right| \leq \sum_{k \geq N} \sum_{1 \leq i \leq j \leq d}\left|\mathbb{E} X_{0}^{i} X_{k}^{j}\right|$, we can find $N \in \mathbb{Z}_{+}$such that

$$
\sum_{k \in N \mathbb{Z}^{d} \backslash\{0\}} \sum_{1 \leq i \leq j \leq d}\left|\mathbb{E} X_{0}^{i} X_{k}^{j}\right| \leq \frac{\sigma^{2}}{2}
$$

For each $(k, i) \in\{0, N-1\}^{d} \times\{1, \ldots, d\}$, we can define $A_{k, i}=\Lambda \cap\left(\left(k+N \mathbb{Z}^{d}\right) \times\{i\}\right)$. By the pigeon-hole principle, there exists $\left(k_{0}, i_{0}\right)$ such that $\left|A_{k_{0}, i_{0}}\right| \geq \frac{|\Lambda|}{d N^{d}}$. Let $\tilde{X}$ be the $\left|A_{k_{0}, i_{0}}\right|$-dimensional Gaussian vector composed by the $\left(X_{e}\right)_{e \in A_{k_{0}, i_{0}}}$; it is obvious that

$$
\begin{aligned}
\mathbb{P}\left(\sum_{(k, i) \in \Lambda}\left(X_{k}^{i}\right)^{2} \leq|\Lambda| \varepsilon\right) & \leq \mathbb{P}\left(\|\tilde{X}\|_{2}^{2} \leq|\Lambda| \varepsilon\right) \\
& \leq \mathbb{P}\left(\|\tilde{X}\|_{2}^{2} \leq\left|A_{k_{0}, i_{0}}\right| d N^{d} \varepsilon\right) .
\end{aligned}
$$

By Lemma 7.4,

$$
d N^{d} \varepsilon<\Upsilon(C) \Longrightarrow \mathbb{P}\left(\|\tilde{X}\|_{2}^{2} \leq\left|A_{k_{0}, i_{0}}\right| d N^{d} \varepsilon\right) \leq \exp \left(-\left|A_{k_{0}, i_{0}}\right| h\left(\frac{d N^{d} \varepsilon}{\Upsilon(C)}\right)\right)
$$

where the covariance matrix for $\tilde{X}$ is $C=\left(\mathbb{E} X_{k}^{i} X_{l}^{j}\right)_{((k, i),(l, j)) \in A_{k_{0}, i_{0}} \times A_{k_{0}, i_{0}}}$. But

$$
\begin{aligned}
\Upsilon(C) & \geq \inf _{\left(k, i_{0}\right) \in A_{k_{0}, i_{0}}} \mathbb{E}\left(X_{k}^{i_{0}}\right)^{2}-\sum_{\left(l, i_{0}\right) \in A_{k_{0}, i_{0}} ;\left(l, i_{0}\right) \neq\left(k, i_{0}\right)}\left|\mathbb{E} X_{k}^{i} X_{l}^{i_{0}}\right| \\
& \geq \sigma^{2}-\sup _{\left(k, i_{0}\right) \in A_{k_{0}, i_{0}}\left(l, i_{0}\right) \in A_{k_{0}, i_{0} ;} ;\left(l, i_{0}\right) \neq(k, i)}\left|\mathbb{E} X_{k}^{i_{0}} X_{l}^{i_{0}}\right| \\
& \geq \sigma^{2}-\sup _{\left(k, i_{0}\right) \in A_{k_{0}, i_{0}}\left(l, i_{0}\right) \in A_{k_{0}, i_{0} ;\left(l, i_{0}\right) \neq(k, i)}}\left|\mathbb{E} X_{0}^{i_{0}} X_{k-l}^{i_{0}}\right| \\
& \geq \sigma^{2}-\sum_{k \in N \mathbb{Z}^{d} \backslash\{0\}}\left|\mathbb{E} X_{0}^{i_{0}} X_{k}^{i_{0}}\right| \\
& \geq \sigma^{2}-\frac{\sigma^{2}}{2}=\frac{\sigma^{2}}{2} .
\end{aligned}
$$

It follows that

$$
\forall \varepsilon \in\left(0, \frac{\sigma^{2}}{2 N d}\right) \quad \mathbb{P}\left(\sum_{(k, i) \in \Lambda}\left(X_{k}^{i}\right)^{2} \leq|\Lambda| \varepsilon\right) \leq \exp \left(-\frac{1}{d N^{d}} h\left(\frac{2 \varepsilon}{\sigma^{2}}\right)|\Lambda|\right) .
$$

We can now claim that the assumptions of Theorem 4.3 are fulfilled. Since $\lim _{x \rightarrow 0} h(x)=+\infty$, we have $K_{0}=0$. Then, the application $\mu$ associated to $p$ and $S_{\nu}$ is a norm for each $p>p_{c}$.

With the help of Lemma 7.3, it is not difficult to see that

$$
S_{\nu}\left(\eta \in \Omega_{S} ; \sum_{e \in \Lambda} \eta_{e} \geq|\Lambda| a^{2}\right) \leq \mathrm{e}^{-|\Lambda| h\left(\frac{a^{2}}{S}\right)}
$$

holds for each $a>S$. It follows that $\left(H_{\alpha}\right)$ holds for each $\alpha>1$, and hence that the assumptions of Theorem 5.3 are fulfilled. 


\subsection{Cost and speed for road networks}

Imagine that there exist $n$ companies $\left(C_{i}\right)_{1 \leq i \leq n}$ that want to build roads on the same $\mathbb{Z}^{d}$ lattice: company $C_{i}$, independently from the others, tries to build a road on edge $e$ with probability $p_{i}$ (set then $\omega_{e, i}=1$ ) and leaves the edge $e$ empty with probability $1-p_{i}$ (set then $\omega_{e, i}=0$ ), all constructions being independent. When two or more companies want to build a road on the same edge, they share the possession of the road. A client of company $C_{i}$ can only use roads at least partially owned by $C_{i}$. Assume that each $p_{i}>p_{c}(d)$ to ensure that almost surely, company $C_{i}$ has a real (infinite) road network. Portions of roads owned by company $C_{i}$ that are not linked to the infinite cluster cannot be used by its clients. Set, if $e=\{a, b\} \in \mathbb{E}^{d}$ is an edge:

$$
\eta_{e}=\sum_{i=1}^{n} \omega_{e, i} \mathbb{1}_{\left\{\left|C_{i}(a)\right|+\left|C_{i}(b)\right|=+\infty\right\}} .
$$

Then $\eta_{e}$ counts the number of companies that offer the edge $e$ as a possible road for their clients. Now, associate to the edge $e$ a random value:

where $f$ is a deterministic function.

$$
\eta_{e, i}=f\left(i, \eta_{e}\right)
$$

For instance, we can imagine that $f\left(i, \eta_{e}\right)$ represents the costs for company $C_{i}$ to build a road on the edge $e$ : these costs depend on the specificity of company $C_{i}$ (that is why $f$ depends on $i$ ), and also on the total number $\eta_{e}$ of companies that want to build a road on th edge $e$ (they can share the costs of construction for instance). In this case, the function $f$ is decreasing in the second variable.

Another example can be the following: $f\left(i, \eta_{e}\right)$ represents the time needed for clients of company $C_{i}$ to cross the edge $e$ : the larger the number of companies that own this edge is, and the larger the number of users to take this portion will be, and the longer the time needed to cross the edge will be. In this case, the function $f$ does not depend on $i$ and is increasing in the other variable.

The next result gives the existence of an asymptotic shape in this context. In other words, the travel times for a client of $C_{i}$ will give rise to a deterministic asymptotic shape that does not depend on the random environment (neither his company road network, nor the other networks), but only on the densities $\left(p_{i}\right)_{1 \leq i \leq n}$ of the distinct networks and on the function $f$. The same result is of course available for the cost point of view.

Theorem 7.5. Let $\Omega=\left(\Omega_{E}\right)^{n}$ and $\mathbb{P}=\mathbb{P}_{p_{1}} \otimes \mathbb{P}_{p_{2}} \cdots \otimes \mathbb{P}_{p_{n}}$, with $p_{i}>p_{c}$ for each $i \in\{1, \ldots, n\}$ and $f:\{1, \ldots, n\} \times\{0, \ldots, n\} \rightarrow \mathbb{R}_{+}$with $f(i, n)>0$ as soon as $n \neq 0$. We say that two vertices $k$ and $l$ of $\mathbb{Z}^{d}$ are $i$-connected if there is a path between $k$ and $l$ such that $\omega_{e, i}=1$ for each bond $e$ which is used in the path. For $x \in \mathbb{Z}^{d}$, we denote by $C_{i}(x)$ the $i$-connected component of $x$. Let us also define for $e=\{a, b\} \in \mathbb{E}^{d}$ :

$$
\eta_{e}=\sum_{i=1}^{n} \omega_{e, i} \mathbb{1}_{\left\{\left|C_{i}(a)\right|+\left|C_{i}(b)\right|=+\infty\right\}} .
$$

As before, for $(\omega, \eta) \in \Omega \times \Omega_{S}$, and $(x, y) \in \mathbb{Z}^{d} \times \mathbb{Z}^{d}$, we define the travel time $d_{i}(x, y)(\omega, \eta)$ to be

$$
\inf _{\gamma} \sum_{e \in \gamma} f\left(i, \eta_{e}\right)
$$

where the infimum is taken on the set of paths whose extremities are $x$ and $y$ and that are $i$-open in the configuration $\omega$. For $t \geq 0$ and $i \in\{1, \ldots, d\}$, we note

$$
B_{t}^{i}=\left\{k \in \mathbb{Z}^{d} ; d_{i}(0, k) \leq t\right\} .
$$

For $i \in\{1, \ldots, d\}$, let us note by $\overline{\mathbb{P}}_{i}$ the probability measure defined by

$$
\overline{\mathbb{P}}_{i}(A)=\frac{\mathbb{P}\left(A \cap\left\{\left|C_{i}(0)\right|=+\infty\right\}\right)}{\mathbb{P}\left(\left|C_{i}(0)\right|=+\infty\right)} .
$$


Then, there is a convex compact set $A^{i}$ with non-empty interior such that

$$
\lim _{t \rightarrow+\infty} \frac{B_{t}^{i}}{t}=A^{i} \quad \overline{\mathbb{P}}_{i} \text { a.s. }
$$

where the convergence holds for Hausdorff's topology.

Proof. Fix $i \in\{1, \ldots, n\}$. For $e=\{a, b\} \in \mathbb{E}^{d}$, let us define

$$
\eta_{e}^{*}=f\left(i, 1+\sum_{j \in\{1, \ldots, n\} \backslash\{i\}} \omega_{e, j} \mathbb{1}_{\left\{\left|C_{j}(a)\right|+\left|C_{j}(b)\right|=+\infty\right\}}\right)
$$

and the travel time $d^{*}(x, y)(\omega, \eta)$ to be

$$
\inf _{\gamma} \sum_{e \in \gamma} \eta_{e}^{*}
$$

where the infimum is taken on the set of paths whose extremities are $x$ and $y$ and that are $i$-open in the configuration $\omega$. For $t \geq 0$, we note

$$
B_{t}^{*}=\left\{k \in \mathbb{Z}^{d} ; d^{*}(0, k) \leq t\right\} .
$$

It is obvious that

$$
\overline{\mathbb{P}}_{i}\left(\forall t \geq 0 \quad B_{t}^{i}=B_{t}^{*}\right)=1 .
$$

Since the passage time $\left(\eta_{e}^{*}\right)_{e \in \mathbb{E}^{d}}$ are independent from the environment $\left(\omega_{k, i}\right)_{k \in \mathbb{Z}^{d}}$, it will be possible to use the preceding theorems.

The first step is to check that the law of $\left(\eta_{e}^{*}\right)_{e \in \mathbb{E}^{d}}$ is shift-invariant and ergodic under the translations of $\mathbb{Z}^{d}$. Obviously

$$
\underset{j \in\{1, \ldots, n\} \backslash\{i\}}{\otimes} \mathbb{P}_{p_{j}}=\left(\underset{j \in\{1, \ldots, n\} \backslash\{i\}}{\otimes}\left(1-p_{j}\right) \delta_{0}+p_{j} \delta_{1}\right)^{\otimes \mathbb{E}^{d}}
$$

is shift-invariant and ergodic under the translations of $\mathbb{Z}^{d}$. For simplicity, we note $L=\left(\underset{j \in\{1, \ldots, n\} \backslash\{i\}}{\otimes} \delta_{0}+\right.$ $\left.p_{j} \delta_{1}\right)^{\otimes \mathbb{E}^{d}}$. We note

$$
F(\omega)=\left(f\left(i, 1+\sum_{j \in\{1, \ldots, n\} \backslash\{i\}} \omega_{e, j} \mathbb{1}_{\left|C_{j}(a(e))\right|+\left|C_{j}(b(e))\right|=+\infty}\right)\right)_{e \in \mathbb{E}^{d}},
$$

with the notation $e=\{a(e), b(e)\}$.

The law of $\left(\eta_{e}^{*}\right)_{e \in \mathbb{E}^{d}}$ is precisely the image of $L$ by the transformation $F$. Let us denote by $S_{\nu}$ this law. It is easy to see that $F \circ \theta_{u}=\theta_{u} \circ F$ holds for each $u \in \mathbb{Z}^{d}$, so we have the commuting diagram:

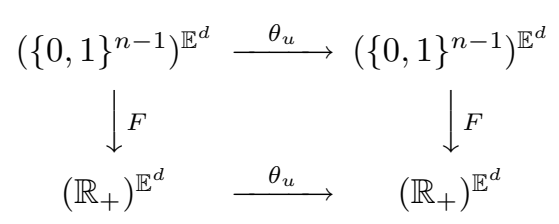


For any event $A \in \mathcal{B}\left(\left(\mathbb{R}_{+}\right)^{\mathbb{E}^{d}}\right)$, we have

$$
\begin{aligned}
S_{\nu}\left(\theta_{u}^{-1}(A)\right) & =L\left(F^{-1}\left(\theta_{u}^{-1}(A)\right)\right. \\
& =L\left(\left(\theta_{u} \circ F\right)^{-1}(A)\right) \\
& =L\left(\left(F \circ \theta_{u}\right)^{-1}(A)\right) \\
& =L\left(\theta_{u}^{-1}\left(F^{-1}(A)\right)\right. \\
& =L\left(F^{-1}(A)\right)=S_{\nu}(A) .
\end{aligned}
$$

Then, $S_{\nu}$ is invariant under the translations.

It is now clear that the dynamical system $\left(\left(\mathbb{R}_{+}\right)^{\mathbb{E}^{d}}, \mathcal{B}\left(\left(\mathbb{R}_{+}\right)^{\mathbb{E}^{d}}\right), S_{\nu}, \theta_{u}\right)$ is a factor of the dynamical system $\left(\left(\{0,1\}^{n-1}\right)^{\mathbb{E}^{d}}, \mathcal{B}\left(\left(\{0,1\}^{n-1}\right)^{\mathbb{E}^{d}}\right), L, \theta_{u}\right)$. Since the dynamical system $\left(\left(\{0,1\}^{n-1}\right)^{\mathbb{E}^{d}}, \mathcal{B}\left(\left(\{0,1\}^{n-1}\right)^{\mathbb{E}^{d}}\right), L, \theta_{u}\right)$ is ergodic, it follows that the dynamical system $\left(\left(\mathbb{R}_{+}\right)^{\mathbb{E}^{d}}, \mathcal{B}\left(\left(\mathbb{R}_{+}\right)^{\mathbb{E}^{d}}\right), S_{\nu}, \theta_{u}\right)$ is ergodic too - see for instance proposition 1.7 of Brown [4].

Now, since $\min f(i, 1+)>$.0 and $\max f(i, 1+)<.+\infty$, the conclusion follows from the remark that we have done before the proof of Theorem 5.3.

\section{REFERENCES}

[1] M. Aizenman, H. Kesten and C.M. Newman, Uniqueness of the infinite cluster and continuity of connectivity functions for short and long range percolation. Comm. Math. Phys. 111 (1987) 505-531.

[2] P. Antal and A. Pisztora, On the chemical distance for supercritical Bernoulli percolation. Ann. Probab. 24 (1996) $1036-1048$.

[3] D. Boivin, First passage percolation: the stationary case. Probab. Theory Related Fields 86 (1990) 491-499.

[4] J.R. Brown, Ergodic theory and topological dynamics. Academic Press, Harcourt Brace Jovanovich Publishers, New York. Pure Appl. Math. 70 (1976).

[5] R.M. Burton and M. Keane, Density and uniqueness in percolation. Comm. Math. Phys. 121 (1989) 501-505.

[6] J.T. Cox, The time constant of first-passage percolation on the square lattice. Adv. Appl. Probab. 12 (1980) 864-879.

[7] J.T. Cox and R. Durrett, Some limit theorems for percolation processes with necessary and sufficient conditions. Ann. Probab. 9 (1981) 583-603.

[8] J.T. Cox and H. Kesten, On the continuity of the time constant of first-passage percolation. J. Appl. Probab. 18 (1981) 809-819.

[9] R. Durrett and T.M. Liggett, The shape of the limit set in Richardson's growth model. Ann. Probab. 9 (1981) $186-193$.

[10] O. Garet, Percolation transition for some excursion sets. Electron. J. Probab. 9 (2004) 255-292 (electronic).

[11] O. Häggström and R. Meester, Asymptotic shapes for stationary first passage percolation. Ann. Probab. 23 (1995) 1511-1522.

[12] J.M. Hammersley and D.J.A. Welsh, First-passage percolation, subadditive processes, stochastic networks, and generalized renewal theory, in Proc. Internat. Res. Semin., Statist. Lab., Univ. California, Berkeley, Calif., Springer-Verlag, New York (1965) 61-110.

[13] H. Kesten, Aspects of first passage percolation, in École d'été de probabilités de Saint-Flour, XIV-1984, Springer, Berlin. Lect. Notes Math. 1180 (1986) 125-264.

[14] H. Kesten and Y. Zhang, The probability of a large finite cluster in supercritical Bernoulli percolation. Ann. Probab. 18 (1990) $537-555$.

[15] R. Marchand, Strict inequalities for the time constant in first passage percolation. Ann. Appl. Probab. 12 (2002) $1001-1038$.

[16] D. Richardson, Random growth in a tessellation. Proc. Cambridge Philos. Soc. 74 (1973) 515-528.

[17] Y.G. Sinai, Introduction to ergodic theory. Princeton University Press, Princeton, N.J., Translated by V. Scheffer. Math. Notes 18 (1976).

[18] W.F. Stout, Almost sure convergence. Academic Press, A subsidiary of Harcourt Brace Jovanovich, Publishers, New YorkLondon. Probab. Math. Statist. 24 (1974).

[19] J. van den Berg and H. Kesten, Inequalities for the time constant in first-passage percolation. Ann. Appl. Probab. 3 (1993) $56-80$. 